\title{
The Design of Post-Grant Patent Challenges
}

\author{
Jing-Yuan Chiou*
}

January 2009

\begin{abstract}
This paper proposes a patent challenge mechanism with partial patent rights previously granted to the patent-holder as the challenge reward. Transferring patent rights to a successful challenger raises the incentive to search for patent-defeating prior art, and, after the discovery of the information, helps deter collusion between the patent-holder and the challenger. It also reduces costly opportunistic patenting and therefore improves patent application quality. However, from an ex post point of view, over-search ensues when the collusion problem is severe. The optimal re-allocation of patent rights, then, calls for a careful balance between these costs and benefits.
\end{abstract}

Keywords: Collusion, Patent Challenge, Patent Quality.

JEL codes: K40, O31, O34.

\section{Introduction}

The patent system, established to encourage innovation, involves costs and benefits. It aims to strike a balance between losses associated with temporary monopolies and gains from long-term technological progress (Nordhaus, 1969; Green and Scotchmer,

${ }^{*}$ Assistant Professor, IMT Lucca. This is the first chapter of my Ph.D. dissertation submitted to Universitè de Toulouse 1. I would like to thank Bernard Caillaud, Vincenzo Denicolò, Josh Lerner, Simon Ma, Richard Schmidtke, Jean Tirole, and John Turner for useful comments. Special thanks go to Jean Tirole for his continuing support and encouragement. Feedback from participants of seminars and conferences at Toulouse, IMT Lucca, NBER, South Carolina, UCL, Pompeu Fabra, IESE, Heriot-Watt, IIOC 2006, FEMES 2006, and Manchester IP and IT workshop are appreciated. All errors are mine. Comments are welcome and please send to: jy.chiou@imtlucca.it 
1995). However, if a patented technology turns out to be 'old,' i.e., already in the public domain, losses come without compensating benefits. For a patent system to serve social interests, it is essential to maintain the good quality of issued patents, in terms of patentability requirements mandated by the patent law. ${ }^{1}$ In the United States and other major economies, this task primarily falls on the patent office, though the private sector also plays an important role.

Among various advantages, private players may have better information than a public agency (Lemley, 2001). For instance, technology-intensive firms may have a comparative advantage in assessing the value of an invention, and therefore identifying those patent applications worthy of detailed examination. Professional researchers and amateur garage inventors may be more familiar with the state of the art and know where to 'dig' in the public domain to discover the prior art. This information forms the basis to determine whether an invention represents enough technological advancement, relative to current knowledge, to justify patent protection. To better exploit this reservoir of knowledge, a properly designed patent challenge system is indispensable for a third party to submit useful information and challenge the validity of an issued patent.

This paper proposes a special challenge mechanism, that is, the re-allocation of patent rights upon the disclosure of patent-defeating prior art. It asks: After a challenger presents the information, how much reward should be given to the challenger in the form of patent rights previously granted to the patent-holder, to what extent can society withdraw issued patent rights, and should the patent-holder be left with some residual rights?

Our analysis addresses two incentive problems: Incentives to look for the prior art and collusion associated with patent challenges. When the prior art is discovered, most existing procedures mandate the revoking of the patent rights in its entirety. Since a patent grants monopoly access to an invention, it generates the highest benefit one can extract from technology users. This benefit gives the challenger a strong incentive to strike a deal with the patent-holder rather than invalidate the patent and dissipate monopoly rent (Thomas, 2001; Hovenkamp et al., 2003; Miller, 2003). ${ }^{2}$

\footnotetext{
${ }^{1}$ The three fundamental requirements in most jurisdictions are novelty, non-obviousness (also called 'inventive step' in Europe), and usefulness ('industrial applications' in Europe).

${ }^{2}$ In the past decade the Federal Trade Commission has consistently challenged 'reverse payment' in the pharmaceutical industry on the ground that it serves an anticompetitive purpose where a brand-name drug maker colludes and persuades a generic drug maker to stay off the
} 
Regarding incentives to search for information, the mere fact that the prior art is not readily available to patent examiners suggests a non-negligible search cost, which raises concerns about whether private search incentives align with social interests.

We start the analysis with the ex post stage (after the patent is granted). Our results suggest that the optimal post-challenge allocation of patent rights responds to the two incentive issues in a non-trivial and interesting way. In particular, the socially optimal level of information search is affected by the extent of collusion (Section 3). Collusion deterrence can be achieved by fully depriving the original patent-holder of her patent rights, and giving enough reward to the challenger in order to induce prior art submission, with the amount reflecting the degree of collusion, i.e., the extent to which the patent-holder and challenger can realize their joint interests. For instance, when twenty years of monopoly rights are attached to a valid patent, and a successful challenge will cause full withdrawal from the patent-holder, the latter is willing to pay up to twenty-year monopoly rent for the challenger's silence. Given this stake, and assuming for simplicity that the challenger has no intrinsic interests in the technology, ${ }^{3}$ let us consider two polar cases.

First suppose that the patent-holder has some (exogenous) difficulty getting the challenger on board. For example, the challenger is 'incorruptible' and heavily discounts any bribery received from the patent-holder, or it is difficult to organize a side collusive transaction. In this case, a minor reward, say, one year of monopoly rights, would be sufficient to deter collusion, and so a successful challenge would bring a social benefit of nineteen years' reduction on the monopoly loss. When the challenger does not fully internalize this sizable benefit, there is a classical under-search problem. An additional reward to the challenger may be necessary to encourage information search.

If, by contrast, collusion is 'perfect' such that the challenger and patent-holder can coordinate to realize their maximal joint profit, then the whole twenty years rights have to be given to the challenger to prevent collusion. In other words, ex post the monopoly rights will not be reduced, whether there is a challenge or not. A successful challenge only causes a transfer of the whole twenty rights from one hand to the other, and any information search, from an ex post point of view, is a pure rent-seeking activity and

market by virtue of the latter's ability to strike down or invent-around the patent covering the targeted brand-name drug. See the statement of Commissioner J. Leibowitz, prepared before the Special Committee on Aging of the U.S. Senate on July 20, 2006 (available online at http://www.ftc.gov/os/2006/07/P052103BarrierstoGenericEntryTestimonySenate07202006.pdf.)

${ }^{3}$ In later discussion we consider a more complicated case where the challenger is also a use of the patented technology. The insight, however, remains the same. 
thus socially wasteful. To overcome this over-search problem, in this extreme case the optimal policy should treat the original patent-holder leniently and leave all patent rights in the hands of the original owner, and no challenge should take place.

Despite the ex post cost of over search, patent challenges may deliver some ex ante benefits. In Section 4 we show that they can discipline patent applicants' opportunistic behavior and raise patent quality. A sound policy, then, calls for a careful balance between ex post rent-seeking costs and ex ante benefits of higher patent quality.

To conclude the paper, in Section 5 we discuss some implementation issues as well as future research. We first argue why employing this mechanism would improve the current situation, and why we are in favor of using patent rights as a challenge reward as opposed to, say, a monetary reward. Because of the critical role the extent of collusion plays in shaping the optimal mechanism, we also offer some ideas about how to measure this parameter, with respect to the market structure and patent quality, respectively.

Related literature and reform proposals: The United States Patent and Trademark Office (USPTO) has been repeatedly criticized for issuing low quality patents. ${ }^{4}$ With dubious quality control at the USPTO, it is troublesome to observe some challenger-unfriendly features of the U.S. post-grant challenge system, patent re-examination, and the fact that there are relatively few re-examination requests. ${ }^{5}$ Compared with its European counterpart, patent opposition, the U.S. system significantly restricts the issues that can be raised in a challenge, limits the extent to which a challenger can participate in the procedure, and limits appeals. ${ }^{6}$ Accordingly, a number

\footnotetext{
${ }^{4}$ Two 'you can patent that!?' examples are "Method of executing a tennis stroke," U.S. patent 5,993,336, and "Method of swinging on a swing," U.S. patent 6,368,227. The swing patent is granted on April 2002 to a five-year-old son of a patent lawyer. A less amusing example is Amazon's "one-click" patent, U.S. patent 5,960,411, which helped Amazon win a preliminary injunction against its major competitor, Barnes \& Noble, just before the 1999's Christmas season. Statistical studies present similar concerns. Quillen and Webster (2001) shows that, after taking into account the continuation application and continuation-in-part applications, the USPTO's allowance rate (the number of applications allowed divided by the number filed) in the mid-1990s is $95 \%$, compared to $68 \%$ and $65 \%$ for the European and Japanese patent offices, respectively. Farrell and Shapiro (2008) recently argue that low quality patents do have significant economic impact.

${ }^{5}$ Graham et al. (2003) reports that only $0.3 \%$ of patents granted between 1991-8 are reexamined and half of the requests are brought by holders of the patent.

${ }^{6}$ In the U.S., a reexamination request can only be based on the ground of prior art citation, i.e., whether the patent office has missed an important prior art in issuing the patent. In Europe, an opposition can be filed on issues as diverse as the subject matter, disclosure requirement, etc. For challenger participation, an inter partes reexamination was introduced by the 1999 legislature: The American Inventors Protection Act. Before that, U.S. procedure was solely ex parte; a challenger's role was confined to the submission of
} 
of reform proposals suggest several procedural changes to the U.S. system, including introducing an alternative timing, i.e. pre-grant challenges, broadening the grounds on which to initiate a challenge, and streamlining current procedures to ensure cost efficiency and timeliness, to name a few (FTC, 2003; NAS, 2004; Hall and Harhoff, 2004; Jaffe and Lerner, 2004). ${ }^{7}$ These proposals have been incorporated into the Patent Reform Act of 2007 introduced into both the U.S. Senate and House of Representatives. ${ }^{8}$

Although these procedural reforms deserve serious consideration, their effect would be constrained by a challenger's intrinsic incentives in both information search and patent challenges. Recognizing this limitation, legal scholars have proposed to construct a bounty system to provide a potential challenger with extra monetary incentives. Thomas (2001) suggests an examination-stage bounty paid by the patent applicant to a 'whistleblower.' Miller (2003) favors a bounty at the litigation stage, and suggests rewarding the challenger with the patentee's past profits, which, as we shall see, has a flavor similar to our approach. ${ }^{9}$ In fact, bounties have already been initiated by private efforts. Around 2001-2002, BountyQuest was launched online to serve as a platform to post and respond to bounties for patent-defeating information. ${ }^{10}$ After a few years in existence, it does not seem to work well, and no competing firm has appeared despite widespread complaints about poor patent quality.

Most of the preceding proposals, including the reform bill under discussion, take as granted that a patent is fully invalidated after a successful challenge. By contrast, the Hatch-Waxman Act prescribes a very different regime for the U.S. pharmaceutical industry. Under this act, a successful challenger is rewarded with a 180-day

an initial statement of why a patent should not be granted, together with supporting evidence, while the patent-holder could fully communicate with the patent office. The higher level of participation permitted in inter partes reexamination, however, entails limited means of appeal: issues that are raised, or could have been raised during reexamination cannot be readdressed in later litigation involving the same challenger. By contrast, there is no such restriction in the European opposition, and both the challenger as well as the agency are allowed more active roles. The Opposition Division of the European Patent Office can search for new prior art and pursue cases on its own, even if private parties want to settle and withdraw the case.

7. For a general discussion, see the special issue of Berkeley Technology Law Review (2004) 19(3). Some commentators advocate a shift toward the European opposition process and provide estimations of the benefit the U.S. could realize by adopting the European system (Graham et al., 2003; Graham and Harhoff, 2005; Levin and Levin, 2003).

${ }^{8}$ The House version was sponsored by Representative H. Berman (H.R. 1908) and has passed on Sep. 7, 2007. The Senate version, sponsored by Senator P. Leahy (S. 1145), however, has been taken off the schedule (last checked Nov. 2008).

${ }^{9}$ We provide a preliminary analysis of the bounty system in Appendix E.

${ }^{10}$ Official website: http://bountyquest . master. com (last visit on November 23, 2005). Somewhat amusingly, the founder of BountyQuest applied for a patent on the business model of the company. 
monopoly right to market a generic version of the drug covered by an invalidated patent. Thus, the act provides a successful challenger with a shorter but still positive period of monopoly. ${ }^{11}$ As we are aware of, only Choi (2005) has mentioned applying the Hatch-Waxman scheme to other industries, but doesn't provide a formal analysis. The main contribution of this paper is thus to provide a formal treatment of the Hatch-Waxman type mechanism and examines factors driving its optimal shape. To the best of our knowledge, no previous work has formally addressed this issue, or more generally, the optimal design of a post-grant challenge mechanism.

Another strand of literature considers patent-granting as a two-stage process, which consists of both patent office examination and post-grant private challenges. Scholars put different emphases on either stage and derive different policy implications. Lemley (2001)'s 'rational ignorant' patent office suggests that, due to the private challenger's information advantages discussed above, it would be more cost efficient to rely on private litigation to weed out bad patents rather than reforming the patent office. Contrarily, Farrell and Merges (2004) and Chiou (2008) raise several limitations of private challenges, such as asymmetric stakes and asymmetric information between the patent-holder and challenger. ${ }^{12}$ As a consequence they argue that the problem should be addressed more at the 'upstream', patent examination stage, either the patent office should play a more important role, or the applicant should be given more incentives to help the examination process. Our stance in this paper is that no matter how important the patent office is, it would be unrealistic to expect the patent office to do a perfect job. Private challenges remain an indispensable means of improving patent quality, and thus the design of a challenge mechanism is a worthy topic.

\section{Model}

The authority in charge of patent institutions, namely, Congress or courts, designs challenge policy. Given the patent institution, an inventor $A$ decides whether to un-

\footnotetext{
${ }^{11}$ To be more precise, the 180 -day exclusivity period provides only quasi-monopoly rights. A controversial practice called 'authorized generics' allows brand drug makers to license to other generic firms and compete with the first challenger in the generic market.

${ }^{12}$ Farrell and Merges (2004) suggests that asymmetric stakes may come from the pass-through problem and the public goods nature of a patent challenge, which happens when the patent rights are fully revoked after a successful challenge. Chiou (2008) shows that case selection associated with asymmetric information may lead to litigation against a strong patent, while a weak patent may be settled and escape a court challenge.
} 
dertake a R\&D project; if so, she applies for and is granted a patent, ${ }^{13}$ and then licenses her patent. Technology users $B_{i}, i \in\{1,2, \ldots, N\}, N>1$, decide whether to buy the license. One of the users, $B_{j}$, will be endowed with the information search capacity. He then has to decides whether to search for prior art, and once the information is found, whether to submit it to the patent authority or to collude with the patent-holder. Assume that all private parties are protected by limited liability.

Technology: In the basic model the inventor side is constructed in a simple adverse selection manner. The inventor $A$ undertakes a $\mathrm{R} \& \mathrm{D}$ project with cost $k \in$ $\{0, K\}, K>0$. A positive cost corresponds to a technology breakthrough: Relative to current stock of knowledge, a technology is a true invention if and only if it requires a positive cost, $k=K$. In contrast, if $k=0$, the technology already exists in the public domain. Only the inventor is aware of the true $k$; all other players share belief that $\operatorname{Pr}(k=K)=\alpha \in(0,1)$. We call $A$ an honest (opportunistic) inventor when $k=K$ $(k=0$, respectively).

The technology's value is independent of $k$, though. ${ }^{14}$ User $B_{i}$ values the technology at $v_{i} \in\left\{v^{h}, v^{l}\right\}$, with $v^{h}>v^{l}$, which is private information to $B_{i}$. The $v_{i}$ s are i.i.d. with $\operatorname{Pr}\left(v_{i}=v^{h}\right)=\rho \in(0,1)$. Define $v^{e} \equiv \rho v^{h}+(1-\rho) v^{l}, \pi \equiv \rho v^{h}$, and $d \equiv(1-\rho) v^{l}$. Assume $\pi>v^{l}$, so that the monopoly licensing fee is $v^{h}$, and the ex post inefficiency or deadweight loss associated with monopoly is $d$. In aggregate, define $V^{e} \equiv N v^{e}$, $\Pi \equiv N \pi$, and $D \equiv N d$.

When $k=0$ and so there is no innovation, a piece of patent-defeating prior art (henceforth, prior art) can be found indicating that indeed no patent protection should be granted. Assume the prior art exists if and only if $k=0$, and it is a piece of hard information. When $k=K$, there is no way to forge a non-existing information. But when it exists and has been found, the searcher can withhold the prior art and collude with the patent-holder (Tirole, 1986). In terms of legal requirements of patentability, this is mostly consistent with the novelty criterion, which has a clear definition of what information can be considered as patent-defeating, and the patent examination body's problem is to find, but not interpret the information.

The information structure implies that the patent office errs only by granting bogus patents (those which don't meet patentability criteria). Given existence of prior art,

\footnotetext{
${ }^{13}$ Nothing would be changed if invalid patents were granted with probability less than one.

${ }^{14}$ This captures the idea that some bogus patents, although they do not satisfy the patentability criteria, cover valuable technologies.
} 
the searcher $B_{j}$ can spend a cost $c(\beta)$ to find the information with probability $\beta$, with $c^{\prime}$ and $c^{\prime \prime}>0, c^{\prime \prime \prime} \geq 0, c(0)=c^{\prime}(0)=0$, and $c^{\prime}(1)=\infty$.

Patent protection: We exclude public funds and assume that the patent authority's only policy tool is patent protection, or 'patent power' $\theta \in[0,1]$. When endowed with $\theta$, the patent-holder charges a licensing fee $v^{h}$ and extracts a monopoly rent $\theta \pi$ from each user. User $B_{i}$ then gets $(1-\theta) v_{i}$, and the expected deadweight loss is $\theta d$. A more powerful patent ( $\theta$ higher) rewards the owner with higher profit, at the cost of greater ex post social loss.

To give the patent authority more policy flexibility, we allow for shared ownership of patent rights and assume no efficiency loss from such sharing. If two parties are granted patent protection $\theta^{\prime}$ and $\theta^{\prime \prime}$, with $\theta^{\prime}+\theta^{\prime \prime} \in[0,1]$, they jointly exercise a patent with power $\theta^{\prime}+\theta^{\prime \prime}$.

A straightforward example of a policy instrument is sequential patent rights: One party receives ownership over a period $\left[0, T^{\prime}\right]$, and the other over the period $\left(T^{\prime}, T^{\prime \prime}\right]$, with $\theta^{\prime} \equiv \int_{0}^{T^{\prime}} r e^{-r t} d t$ and $\theta^{\prime \prime} \equiv \int_{T^{\prime}}^{T^{\prime \prime}} r e^{-r t} d t$, where $r>0$ is the interest rate. This instrument perhaps is also the one that could be readily applied across industries.

Alternatively, we could interpret $\theta$ as patent scope. Suppose that for each user $B_{i}$, the patented technology has a continuum of uses distributed on a unit line $[0,1]$. Each use has either high $\left(v^{h}\right)$ or low $\left(v^{l}\right)$ value. For simplicity, assume perfect correlation among uses for the same user. Summing over different uses, the technology is worth either $v^{h}$ or $v^{l}$ to $B_{i}$. Again, this is $B_{i}$ 's private information; others hold the common belief $\rho$. Let the patent scope be a subset of the unit line, with measure $\theta$. For instance, each use corresponds to a patent claim. The patent-holder charges a monopoly price $v^{h}$ over the uses that fall within the scope of the patent, and $B_{i}$ produces without licensing for uses outside the patent scope. The revenue and deadweight loss associated with patent protection then take the same linear form as described above. Shared ownership can be thought of as two parties that are granted patent rights controlling non-overlapping uses, or different patent claims with measure $\theta^{\prime}$ and $\theta^{\prime \prime}$.

To induce innovation, the honest innovator's incentive constraint is

$$
(I C)_{A}: \quad \theta \Pi \geq K \Rightarrow \theta \geq \theta^{*} \equiv \frac{K}{\Pi} .
$$

Assume $0<K \leq \Pi$ and so $\theta^{*} \in(0,1]$. Throughout the paper, the patent system aims at inducing innovation. Absent a challenge, the patent protection should be at least $\theta^{*}$. 


$\begin{array}{cccccc}0 & 1 & 1.5 & 2 & 3 & 4 \\ \perp & \perp & \mid & \mid & \mid & \perp \\ \text { policy } & \begin{array}{l}\text { R\&D and } \\ \text { patenting }\end{array} & \text { search } & \left\{v_{i}\right\} & \begin{array}{l}\text { collusion or } \\ \text { challenge }\end{array} & \text { licensing }\end{array}$

Figure 1: Timing

Referring to Figure 1, the timing of the game is as follows: ${ }^{15}$

- At date 0, the patent authority designs the challenge policy;

- at date $1, A$ decides whether to undertake a $\mathrm{R} \& \mathrm{D}$ project of cost $k$. The patent application and grant follow the expenditure of $k$;

- at date 1.5 , the searcher $B_{j}$ makes a search decision;

- at date 2 , the value of the technology $v_{i}$ is observed by user $B_{i}$;

- at date 3 , when $B_{j}$ has found the prior art, he decides whether to challenge the patent or to collude with the patent-holder; and

- at date 4 , patent rights are allocated and licensing takes place.

We assume that ex ante all users $B_{i}, i \in\{1, \ldots, N\}$, are equally likely to have search capability, and at time 1.5 , one of them, $B_{j}$ is endowed with this capacity. His identity, however, remains unknown to other uses and the patent authority as long as he doesn't initiate a patent challenge. For simplicity, we assume that $B_{j}$ engages in search without learning the true value of $v_{j}$. After learning $v_{j}$, and if $B_{j}$ has found the prior art, he then decides whether to collude with $A$ or to submit the information to the patent authority.

Collusion: In the collusion subgame, the patent-holder is assigned the whole bargaining power and makes a take-it-or-leave-it offer to the challenger. In this offer, $A$ may need to transfer a portion of patent rights (or, equivalently, part of monopoly profit). Following the literature on collusion in organizations, we assume that a side contract is enforceable, but introduce a side transfer efficiency parameter to capture imperfect collusion (Tirole, 1986). If $A$ makes a private transfers $\hat{\theta}$ of patent rights, the recipient receives only $\lambda \hat{\theta}, \lambda \in[0,1]$. The loss $(1-\lambda) \hat{\theta}$ may be due to a probability $1-\lambda$ that such side transfers can be detected; or, to avoid suspicion, the colluding

\footnotetext{
${ }^{15}$ In Appendix $\mathrm{C}$ to $\mathrm{E}$, we consider different variants of the basic model, including: the single downstream user case, $N=1$ (Appendix C); multiple searchers with simultaneous (Appendix D); and the introduction of monetary instruments as well as the possibility of 'type II error,' i.e., the true inventor may be rejected patent protection (Appendix E). Limited liability constraint is also relaxed in the third extension.
} 
parties have to trade in an indirect and less efficient way. Note that limited liability has to be satisfied in this subgame, too. $A$ has no cash to pay as a bribe, and cannot give away more monopoly rights than what she receives from the patent authority.

Assume that all users are subject to the same policy in the absence of a patent challenge. By submitting the prior art $B_{j}$ 's identity is known to the patent authority and so different patent power can be imposed on $B_{j}$ and on other users $B_{-j}$, while all $B_{-j}$ still face the same policy. To shorten the exposition, we restrict the policy space by assuming that, after submission, $B_{j}$ is no longer subject to any licensing obligation. He can freely use the technology and enjoy the whole production surplus $v_{j}$. (In the end of Section 3 we will provide a condition under which this restriction is part of the optimal policy.) A policy, then, is a triplet $\left(\bar{\theta}, \theta^{A}, \theta^{C}\right)$, where $\bar{\theta}$ is the patent right awarded to $A$ when there is no challenge; and when $B_{j}$ submits the prior art, the patent authority reallocates patent rights $\theta^{A}\left(\theta^{C}\right)$ to $A\left(B_{j}\right.$, respectively) against other users $B_{-j}$. Define $S \equiv(N-1) \pi$. Post challenge the inventor $A$ (challenger $B_{j}$ ) garners profit $\theta^{A} S\left(\theta^{C} S\right.$, respectively) from other users $B_{-j}$.

\section{Ex Post Cost of Over-Search}

Two issues arise in the ex post context. When making a search decision, a searcher may not fully internalize all benefits of a patent challenge (the free rider problem). And after discovery, a potential challenger may prefer to collude with the patent-holder and share the monopoly profit extracted from other users, rather than submit prior art to the patent office or court and invalidate the patent (the collusion problem). We assume that private players cannot organize themselves to overcome the free rider problem. ${ }^{16}$ Concerning collusion, in our main analysis we address the optimal policy with full collusion deterrence. ${ }^{17}$ (Appendix B provides a case where this may not hold.)

We first characterize the optimal collusion-free allocation, $\lambda=0$, in order to illustrate the free rider problem. Given a policy $\left(\bar{\theta}, \theta^{A}, \theta^{C}\right)$, the private challenger's optimization program is:

$$
\max _{\beta}[\alpha+(1-\alpha)(1-\beta)](1-\bar{\theta}) v^{e}+(1-\alpha) \beta\left(v^{e}+\theta^{C} S\right)-c(\beta)
$$

\footnotetext{
${ }^{16}$ Note that free riding persists as long as private mechanisms cannot force participation. Another problem is that ex ante it may be difficult to identify potential users.

${ }^{17}$ Appendix D addresses the case of multiple-searcher under collusion-proof mechanisms, where, similar to the patent race literature, the duplication of search cost may also lead to over-search.
} 
When the prior art doesn't exist (with probability $\alpha$ ) or when it exists but $B_{j}$ fails to find it (with probability $(1-\alpha)(1-\beta)$ ), $B_{j}$ faces $A$ 's patent with power $\bar{\theta}$. When $B_{j}$ succeeds in finding the information (with probability $(1-\alpha) \beta$ ), by assumption he is exempted from any licensing obligation and can enjoy the whole production surplus $v^{e}$, plus the additional reward $\theta^{C}$ exerted against other technology users. The privately optimal level of search intensity, $\beta^{s}$, is determined by:

$$
(1-\alpha)\left(\bar{\theta} v^{e}+\theta^{C} S\right) \equiv c^{\prime}\left(\beta^{s}\right)
$$

Consider a policy resembling the status quo situation: $\left(\bar{\theta}, \theta^{A}, \theta^{C}\right)=\left(\theta^{*}, 0,0\right)$, that is, no one gets any monopoly rights after a successful challenge. Under this policy, the social benefit of patent invalidation is the saving of the aggregate monopoly deadweight loss, $\theta^{*} D$, but the private search incentive is driven by a benefit of $\theta^{*} v^{e}$. Under-search ensues whenever $D>v^{e}$, or, $N-1>(\pi / d)$. To encourage search it may be optimal to give $B_{j}$ an extra reward, $\theta^{C}>0$. The following proposition gives the exact condition. (All proofs are relegated to Appendix A.)

Proposition 1. (Collusion-free allocation). Without collusion concerns, the optimal patent protection is $\bar{\theta}=\theta^{*}$ when there is no patent challenge; and post-challenge the patent-holder receives $\theta^{A}=0$, and the challenger receives a positive reward if there is a serious under-search problem and a reward can significantly boost private search, i.e., the optimal $\theta^{C}>0$ if

$$
\left.\theta^{*}[(N-1) d-\pi] \frac{\partial \beta^{s}}{\partial \theta^{C}}\right|_{\bar{\theta}=\theta^{*}, \theta^{C}=0}>\beta_{0}^{s}(N-1) d,
$$

where $\beta_{0}^{s}$ is the privately optimal search intensity evaluated at $\bar{\theta}=\theta^{*}$ and $\theta^{C}=0$.

Starting from $\theta^{C}=0$, a marginal increase of $\theta^{C}$ entails deadweight loss proportional to $\beta_{0}^{s}(N-1) d$, but society benefits from a more intensive search $\left(\partial \beta^{s} / \partial \theta^{C}>0\right)$. To balance the two effects, condition (2) says that the optimal policy calls for $\theta^{C}>0$ when the under-search problem is severe $\left(\beta_{0}^{s}\right.$ low), or when the incentive effect is significant $\left(\partial \beta^{s} / \partial \theta^{C}\right.$ large).

Collusion-proof challenge mechanism: Recall that in collusion subgame the patent-holder $A$ makes an enforceable take-it-or-leave-it offer. The analysis of optimal side contracting is significantly simplified by the restriction that, after a challenge, $B_{j}$ can freely use the technology. It implies that $A$ can only induce $B_{j}$ 's cooperation by promising him a portion of the monopoly rent levied on other uses $B_{-j}$. Collusion 
gains flow from the higher profit collected from $B_{-j}$. Given $\lambda \in(0,1]$ and patent policy $\left(\bar{\theta}, \theta^{A}, \theta^{C}\right)$, define

$$
\Delta \theta \equiv \lambda\left(\bar{\theta}-\theta^{A}\right)-\theta^{C}
$$

which is the gains of trade from collusion. The maximal bribe $A$ is willing to offer is $\bar{\theta}-\theta^{A}$, which has to be discounted by $\lambda$ to reflect the amount transferable to $B_{j}$; while $B_{j}$ must get at least $\theta^{C}$ to participate in collusion. Assume that $A$ has incentives to make the collusive offer only when it is strictly better to do so. The following lemma derives the optimal side contract.

Lemma 1. (Optimal side contract). Collusion takes place if and only if there are gains from doing so, $\Delta \theta>0$. When $\Delta \theta>0$, in the optimal side contract $B_{j}$ receives a free license.

Because side transfer of patent rights entails an efficiency loss $(\lambda \leq 1)$, to economize on its use, $A$ optimally grants $B_{j}$ a free license and leaves him the whole production surplus $v_{j}$. This in turn implies that the patent authority can fully replicate the optimal side contract, thus the collusion-proofness principle holds.

Lemma 2. (Collusion-proofness principle). The patent authority optimally deters collusion in the multiple-user, single-challenger case.

Now we can write down the patent authority's optimization program. ${ }^{18}$

$$
\begin{aligned}
(\mathcal{P})^{s}: \max _{\bar{\theta}, \theta^{A}, \theta^{C}} & \mathcal{W}^{s}=\alpha\left(V^{e}-\bar{\theta} D-K\right)+(1-\alpha)\left\{\left(1-\beta^{s}\right)\left(V^{e}-\bar{\theta} D\right)\right. \\
& \left.+\beta^{s}\left[V^{e}-\left(\theta^{A}+\theta^{C}\right)(N-1) d\right]\right\}-c\left(\beta^{s}\right) \\
\text { s.t. } \quad & (I C)_{A}: \bar{\theta} \geq \theta^{*}, \quad(C P): \Delta \theta \leq 0, \\
& (I C)_{C}: c^{\prime}\left(\beta^{s}\right) \equiv(1-\alpha)\left(\bar{\theta} v^{e}+\theta^{C} S\right) .
\end{aligned}
$$

The patent authority faces three constraints: To encourage innovation the true inventor's incentive constraint $(I C)_{A}$ has to be satisfied; by Lemma 2 collusion should be deterred in this case, thus constraint $(C P)$; and the challenger's search intensity is dictated by his private incentives as described in $(I C)_{C}$.

\footnotetext{
${ }^{18}$ Lemma 2 still holds if $B_{j}$ 's search decision is made after learning the private value $v_{j}$. In fact, the policy restriction that $B_{j}$ automatically gets a free license makes his private information irrelevant. The only difference introduced by this alternative timing is that $B_{J}$ 's optimal search intensity is contingent on $v_{j} \in\left\{v^{l}, v^{h}\right\}$ : for $t \in\{l, h\}, c^{\prime}\left(\beta_{t}^{s}\right) \equiv(1-\alpha)\left(\bar{\theta} v_{t}+\theta^{C} S\right)$. This would complicate the analysis without providing further insight.
} 
When these constraints are satisfied, the true inventor will engage in innovation, which creates a total value of $V^{e}$, but has to be subtracted from innovation cost $K$ and deadweight loss of patent protection $\bar{\theta} D$. On the other hand, when the technology is already in the public domain, without a challenge the society suffers from unwarranted deadweight loss $\bar{\theta} D$, and with the help of a patent challenge this loss can be reduced to $\left(\theta^{A}+\theta^{C}\right)(N-1) d$, for the challenger will realize full production surplus.

Let $\mu$ be the Kuhn-Tucker multiplier of the $(C P)$ constraint, and define

$$
\psi \equiv\left(\bar{\theta}-\theta^{A}-\theta^{C}\right)(N-1) d-\left[\bar{\theta}+\theta^{C}(N-1)\right] \pi,
$$

which reflects the discrepancy between social and private incentives to search. There is over-search (under-search) if $\psi<0$ ( $\psi>0$, respectively).

When $N$ and $\alpha$ are large enough, the optimal $\bar{\theta}=\theta^{*}$. Increasing $\bar{\theta}$ beyond $\theta^{*}$ creates more deadweight loss, strengthens incentives to collude, and encourages private search. But even with under-search, if $\alpha$ is not too small, raising $\theta^{C}$ is a more efficient way to encourage private search.

To determine the optimal $\theta^{A}$ and $\theta^{C}$, as $\lambda$ increases from zero to one, there may exhibit three regimes. First note that from $\lambda \leq 1$, to deter collusion with the least social cost the patent authority should re-allocate the patent rights only to $B_{j}$, with $\theta^{C}=\lambda \theta^{*}$ and $\theta^{A}=0$. However, this allocation rule may be disturbed by inadequate private search incentives. Referring to Figure 2, there exist $\hat{\lambda}$ and $\bar{\lambda}$, with $\hat{\lambda}<\bar{\lambda}$, and possibly $\underline{\lambda}<\hat{\lambda}$, such that:

(i) Encouraging search: when $\lambda \leq \underline{\lambda}$ (if $\underline{\lambda}$ exists), the optimal $\theta^{A}=0$ and $\theta^{C}>\lambda \theta^{*}$. In this range $\psi$ is positive and large, i.e., there is a serious under-search issue, and so a reward of $\lambda \theta^{*}$ doesn't give $B_{j}$ enough search incentives, relative to the social benefit. As in Proposition 1, it may be desirable to further reward $B_{j}, \theta^{C}>\lambda \theta^{*}$; $(C P)$ slacks. This regime may not exist for doing so entails a social cost proportional to $(N-1) d$;

(ii) binding collusion-proofness: when $\lambda \in(\underline{\lambda}, \bar{\lambda}]$, the optimal $\theta^{A}=0$ and $\theta^{C}=$ $\lambda \theta^{*} . B_{j}$ receives the collusion-deterrence reward $\lambda \theta^{*}$. As $\lambda$ increases, reduction of social loss, $\left(\theta^{*}-\theta^{C}\right)(N-1) d$, shrinks, while $B_{j}$ 's search intensity increases. When $\lambda$ is large enough $(\lambda>\hat{\lambda}), \psi$ becomes negative and there is over-search. But as long as the problem of suboptimal private search intensity is moderate $(|\psi|$ not too big), the patent authority sticks to this regime;

(iii) collusion-deterrence cum discouraging search: when $\lambda>\bar{\lambda}$, the optimal 


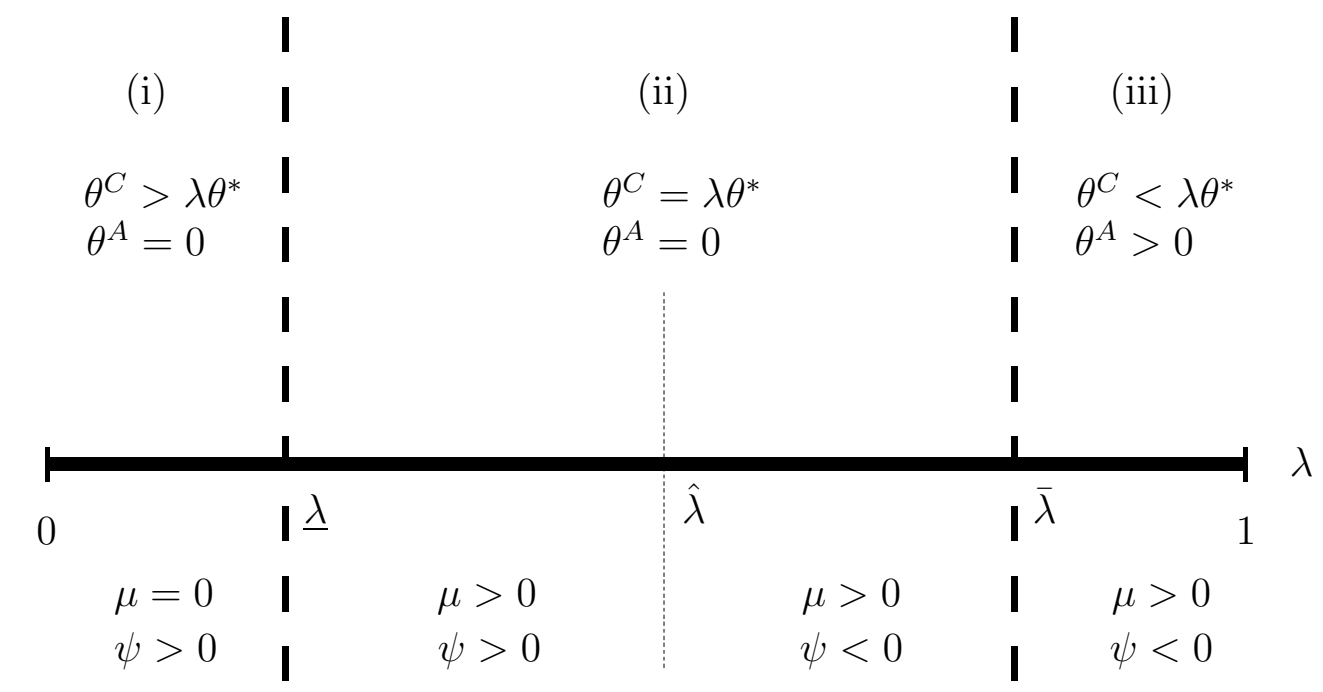

(and significant)

(and significant)

Figure 2: Ex post optimal policy

$0<\theta^{A} \leq \theta^{*}$ and $0 \leq \theta^{C}<\lambda \theta^{*}$, with $\lambda\left(\theta^{*}-\theta^{A}\right)=\theta^{C}$. Rewarding $B_{j}$ with $\lambda \theta^{*}$ leads to very large over-search costs, relative to deadweight loss savings. To dilute $B_{j}$ 's search incentives and at the same time deter collusion, the patent authority leaves part of the patent rights to the inventor $A, \theta^{A}>0$, even after a successful patent challenge.

Proposition 2. (Collusion-proof allocation). Solving the program $(\mathcal{P})^{s}$, after a patent challenge: When the collusion problem is not severe, $\lambda \leq \bar{\lambda}$ (regime (i) and (ii)), the patent-holder is fully deprived of her patent rights, and the challenger is rewarded with partial patent rights; but when $\lambda>\bar{\lambda}$ (regime (iii)), the patent-holder retains (partial) patent rights after a challenge.

Opposed to the collusion-free case, when there is 'perfect collusion,' $\lambda=1$, the total patent rights imposed on $B_{-j}$ cannot be reduced after the patent challenge. In order to satisfy the $(C P)$ constraint, any reduction in $\theta^{A}$ is accompanied with the same amount of increase of $\theta^{C}$. There are no ex post social gains from this re-allocation of patent rights, and private search driven by $\theta^{C}$ is socially wasteful. It is optimal to set $\theta^{A}=\theta^{*}$ and $\theta^{C}=0$. The finding of prior art only increases the social surplus to the extent that $B_{j}$ can freely use the technology in his own production, which is always internalized by $B_{j}$.

Remark. The restriction that a successful challenger is no longer subject to patent 
rights may only change the optimal policy in regime (iii). After a challenge, if $A$ retains some monopoly rights against $B_{j}$, the two may collude even when $\Delta \theta \leq 0$. For $B_{j}$ may be willing to sacrifice $\theta^{C}$ and collude with $A$ in order to gain more operation freedom and enjoy a larger own production surplus. The collusion problem worsens, but the collusion-proofness principle still holds (for in an optimal side offer $A$ grants $B_{j}$ a free license). This policy could enhance welfare only by discouraging private search, a concern only in regime (iii).

Suppose that $\lambda=1$ and $\theta^{A}=\theta^{*}$, and so patent invalidation only concerns $B_{j} .{ }^{19}$ Over-search then ensues: After a challenge $B_{j}$ can freely use the technology and enjoy the whole production surplus $\theta^{*} v^{e}$, but only a portion of this surplus, $\theta^{*} d$, is counted as ex post social benefit, while $\theta^{*} \pi$ is a licensing transfer between the patent-holder and challenger. But if the over-search problem is not too severe, there is no need to provoke further policy adjustment by subjecting $B_{j}$ to $A$ 's patent rights after a challenge. Our restriction then becomes part of the optimal policy.

To derive the formal condition, fix $\bar{\theta}=\theta^{A}=\theta^{*}$, and introduce $\theta_{j}^{A}$ as $A$ 's patent rights against $B_{j}$ after a challenge. The latter's optimal search is determined by

$\hat{\beta}=\arg \max _{\beta}(1-\alpha) \beta\left(\theta^{*}-\theta_{j}^{A}\right) v^{e}-c(\beta) \Rightarrow F O C:(1-\alpha)\left(\theta^{*}-\theta_{j}^{A}\right) v^{e} \equiv c^{\prime}(\hat{\beta})$.

Relevant social welfare consideration is

$$
(1-\alpha)\left[v^{e}-\hat{\beta} \theta_{j}^{A} d-(1-\hat{\beta}) \theta^{*} d\right]-c(\hat{\beta})=(1-\alpha)\left[v^{e}-\theta^{*} d+\hat{\beta}\left(\theta^{*}-\theta_{j}^{A}\right) d\right]-c(\hat{\beta}) .
$$

$B_{j}$ 's challenge only recovers the social loss $\left(\theta^{*}-\theta_{j}^{A}\right) d$. The ptimal $\theta_{j}^{A}=0$ when

$$
\left.\frac{\left(\theta^{*}-\theta_{j}^{A}\right)}{\hat{\beta}} \frac{\partial \hat{\beta}}{\partial\left(\theta^{*}-\theta_{j}^{A}\right)}\right|_{\theta_{j}^{A}=0} \leq \frac{d}{\pi},
$$

where $\hat{\beta}$ is evaluated at $\theta_{j}^{A}=0$.

Remark. After a successful challenge, only in regime (iii) does the optimal mechanism require a sharing of patent rights between the original patent-holder and the challenger. If there is some cost associated with this shared ownership, this regime will become less attractive. In the next section, we discuss another reason why regime (iii) may shrink, namely, the ex ante benefits of patent challenges.

${ }^{19}$ This is similar to the single-user case considered in Appendix C. 


\section{Ex Ante Benefits of Higher Patent Quality}

The previous section concentrates on the ex post role of patent challenges. To fully evaluate the merit of the challenge mechanism, we also need to take into account that patent challenges may improve patent quality (captured by $\alpha$ ) by inducing innovation or by deterring opportunistic patenting in the first place. These ex ante benefits constrain the relevancy of ex post over-search and the associated lenient treatment of the original patent-holder, $\theta^{A}>0$.

Inducing innovation: So far, we have assumed that an honest inventor has no chance to game the system with opportunistic patenting (adverse selection). If, instead, we consider a simple moral hazard element that allows a true inventor to copy and patent a (equally valuable) technology already in the public domain, to induce innovation, the inventor's incentive constraint becomes

$$
\bar{\theta} \Pi-K \geq(1-\beta) \bar{\theta} \Pi+\beta \theta^{A} S \Rightarrow \beta \bar{\theta} \Pi \geq K+\beta \theta^{A} S .
$$

In this case, to encourage innovation requires both carrot and stick. An honest inventor needs to be not only compensated for her R\&D cost, but also kept away from the temptation of opportunistic patenting. A policy $\bar{\theta} \geq \theta^{*}$ fulfills the first purpose and patent challenges serve the second function. That is, a lower payoff following a challenge $\left(\theta^{A} S<\bar{\theta} \Pi\right)$ discourages opportunistic patenting. Note that a higher $\theta^{C}$ enables higher search and challenge intensity, which creates social benefits by reducing the necessary patent protection $\bar{\theta}$ to induce innovation. Also, the appearance of $\theta^{A}$ on the righthand side of condition (3) suggests that a more lenient policy toward the patent-holder $\left(\theta^{A}\right.$ higher $)$ would undermine the incentive power of the patent system. Both effects constrain regime (iii) in Proposition 2.

Deterring opportunistic patenting: Another drawback of the policy $\theta^{A}>0$ is that it may encourage opportunistic patenting. To illustrate this point, we keep the honest inventor's choice set as in the basic model, but modify the opportunistic inventor's choice set as follows: With probability $\alpha^{\prime} \geq 0$, there is a cost $c^{\prime}>0$ to copy the existing technology and prepare for the patent application; and with probability $1-\alpha-\alpha^{\prime}>0$, opportunistic patenting remains costless. If

$$
c^{\prime} \geq \beta \theta^{A} S+(1-\beta) \bar{\theta} \Pi
$$


the portion of opportunistic patenting $\alpha^{\prime}$ can be deterred. This condition is more likely to hold when a patent challenge is more likely to happen ( $\beta$ larger), and the payoff after a challenge is lower ( $\theta^{A}$ smaller).

When condition (4) fails, an issued patent is bogus with probability $1-\alpha$; but when it holds, this probability falls to

$$
\frac{\left(1-\alpha^{\prime}\right)-\alpha}{1-\alpha^{\prime}} \leq 1-\alpha
$$

When $(C P)$ is binding, increasing $\theta^{C}$ yields an additional benefit of a higher patent quality. Again, this concern may increase $\bar{\lambda}$ and shrink regime (iii).

Example. (Two-point search). Consider a simple search technology where $B_{j}$ can find existing prior art with a fixed probability $\beta$ at a cost $c$. Assume also that $\lambda=1$ and so the $(C P)$ constraint is $\theta^{A}+\theta^{C}=\bar{\theta}$. In a linear structure, it suffices to consider two cases, either $\theta^{C}=\bar{\theta}$ or $\theta^{A}=\bar{\theta}$. Assume

$$
(1-\alpha) \beta \bar{\theta} \bar{v}<c \leq\left(1-\frac{\alpha}{1-\alpha^{\prime}}\right) \beta \bar{\theta}(\bar{v}+S) \text { and }(1-\beta) \bar{\theta} \Pi \leq c^{\prime}<\bar{\theta} \Pi
$$

and so search deters costly opportunistic patenting. When $\theta^{C}=\bar{\theta}$ and $\theta^{A}=0, B_{j}$ incurs $c$ to search, but the opportunistic inventor will not spend $c^{\prime}$ to pursue the patent. On the other hand, when $\theta^{A}=\bar{\theta}$ and $\theta^{C}=0, B_{j}$ doesn't search and the opportunistic inventor spends $c^{\prime}$.

The difference in social welfare under the two policies is

$$
\left.\mathcal{W}\right|_{\theta^{C}=\bar{\theta}}-\left.\mathcal{W}\right|_{\theta^{A}=\bar{\theta}}=\alpha^{\prime} \bar{\theta} D+\alpha^{\prime} c^{\prime}-c+\left(1-\alpha-\alpha^{\prime}\right) \beta \bar{\theta} d
$$

Less opportunistic patenting improves social welfare by reducing deadweight loss $\left(\alpha^{\prime} \bar{\theta} D+\right.$ $\left.\left(1-\alpha-\alpha^{\prime}\right) \beta \bar{\theta} d\right)$ and by saving the cost of acquiring a bogus patent $\left(\alpha^{\prime} c^{\prime}\right)$. These benefits are achieved through costly search $(c)$. The deterrence policy $\left(\theta^{C}=\bar{\theta}\right)$ is optimal if the search cost is not too large compared with the cost of opportunistic patenting ( $c-\alpha^{\prime} c^{\prime}$ not large), and the patent quality can be significantly improved ( $\alpha^{\prime}$ high) ${ }^{20} \|$

Proposition 3. (Ex ante benefits). A policy that leaves the original patent-holder some residual rights after a successful challenge, $\theta^{A}>0$, impedes social objectives of encouraging innovation and deterring opportunistic patenting. When these ex ante concerns present, regime (iii) in Proposition 2 shrinks ( $\bar{\lambda}$ increases).

${ }^{20}$ If $\alpha^{\prime}=0,\left.\mathcal{W}\right|_{\theta^{C}=\bar{\theta}}-\left.\mathcal{W}\right|_{\theta^{A}=\bar{\theta}}<0$ and we are in regime (iii). 
By deterring opportunistic patenting and therefore raising the patent application quality, patent challenges have the further benefits of reducing the patent office workload. This in turn may enhance the patent office performance, and create a virtuous circle emphasized by Caillaud and Duchêne (2005) and Jaffe and Lerner (2004).

Remark. (Application fees). When the innovation constraint is characterized by condition (1), the honest inventor receives zero rent under the optimal policy while the opportunistic inventor's expected profit is positive. A uniform application fee here cannot solve the patent quality problem precisely because it fails to distinguish between the two types of applicants (Caillaud and Duchêne, 2005). Plus, after a fee is imposed the patent rights $\bar{\theta}$ should be raised to keep the true inventor's incentive constraint satisfied, which in turn would trigger even higher incentives to collude for the opportunistic patent-holder.

\section{Implementation}

We conclude the paper with some implementation issues. With the exception of the pharmaceutical industry in the U.S., the current situation can be described as a special case in our policy space: $\theta^{A}=\theta^{C}=0$, i.e., a successful challenge destroys all the monopoly rights. This can hardly be optimal whenever there is a danger of collusion, $\lambda>0$. Deterring collusion and encouraging information search then requires extra incentives for the challenger. ${ }^{21}$

More gaming behavior? One might argue that a reward $\theta^{C}>0$ would actually trigger more bogus patent applications, for applicants could later strike down their own patents and enjoy the challenge prize (or arrange someone else to challenge and then share the prize; another form of collusion). This could happen only when an opportunistic player has higher incentives to apply for a patent after we move away from the current system of $\left(\theta^{A}, \theta^{C}\right)=(0,0)$ to $\left(\theta^{A}, \theta^{C}\right) \neq(0,0)$. But a comparison of the opportunistic inventor's payoffs in the two cases shows that the reverse would

\footnotetext{
${ }^{21}$ Even though the determination of the optimal mechanism remains an empirical issue, and conceivably it would be a challenging task to tailor the reward to the theoretically optimal level, we believe that rewarding a challenger with 'mild' patent rights, $0<\theta^{C}<<\bar{\theta}$, would bring significant improvement over current situation. By playing 'safe,' a not-so-large $\theta^{C}$ would avoid the risk of triggering ex post over-search. Even if it cannot solve the collusion problem, this reward would induce more information search, which in turn would undermine the opportunistic patent-holder's rent and thus discourage costly opportunistic patenting as emphasized in Section 4.
} 
be true. To illustrate this, consider the case of Section 3 , and assume $\lambda>0$ so that collusion is a real concern. Under the policy $\left(\theta^{A}, \theta^{C}\right)=(0,0)$, the opportunistic patentee's payoff is $\left(1-\bar{\beta}_{0}^{s}\right) \bar{\theta} \Pi+\bar{\beta}_{0}^{s} \bar{\theta} S$, where $\bar{\beta}_{0}^{s}$ is determined by $(1-\alpha) \bar{\theta} v^{e} \equiv c^{\prime}\left(\bar{\beta}_{0}^{s}\right)$. (Recall that we let the patent-holder keep the whole bargaining power.)

Now, suppose that the ex post optimal collusion-proof mechanism characterized in Proposition 2 is adopted. If the reward $\theta^{C}$ is only granted to the first challenger, the upper bound of the opportunistic $A$ 's payoff is $\left(1-\bar{\beta}_{0}^{s}\right) \bar{\theta} \Pi+\bar{\beta}_{0}^{s}\left(\theta^{A}+\theta^{C}\right) S$. This upper bound is attainable only when $A$ observes whether $B_{j}$ finds the prior art or not, and in the case of discovery, $A$ can grab the total reward $\theta^{A}+\theta^{C}$ by racing faster than $B_{j}$ to the patent office (or retaining someone to do that for her); and knowing that he can never get the reward $\theta^{C}, B_{j}$ 's search intensity remains at $\bar{\beta}_{0}^{s}$. If any of these conditions fail, $A$ 's payoff is strictly smaller than the upper bound. But since $\theta^{A}+\theta^{C} \leq \bar{\theta},{ }^{22}$ the ex post optimal mechanism cannot give a strictly higher return to the opportunistic inventor than when $\left(\theta^{A}, \theta^{C}\right)=(0,0)$. Put differently, if an opportunistic player wants to apply for a patent after we introduce this (properly designed) challenge mechanism, she probably has already done so under current system. ${ }^{23}$

Why not use monetary rewards? Given the necessity of extra challenge incentives, we believe that patent rights is a more appealing reward instrument than bounties, for it demands less information. To deter collusion, the patent authority needs to have an idea of how serious the problem is, i.e., the size of $\lambda$. On top of this, in a bounty scheme, the amount of a monetary reward should also reflect the value of the patented technology $S$, which is composed of both the market size $(N-1)$ and value $(\pi)$ of the patented technology. Setting the bounty too low cannot deter collusion; too high a bounty entails additional cost if it is financed by public funds. Also, limited liability precludes bounties paid by the patent-holder, that is, a pecuniary punishment to opportunistic patenting. A judgment-proof patent-holder is usually an individual inventor or a small firm, or a 'patent troll.'

\footnotetext{
${ }^{22}$ To see this, consider if the collusion-proof constraint is binding, $\lambda\left(\bar{\theta}-\theta^{A}\right)=\theta^{C}$, then $\bar{\theta}=\theta^{A}+\left(\theta^{C} / \lambda\right) \geq$ $\theta^{A}+\theta^{C}$. If it's not binding, then, referring to Figure 2, the optimal $\theta^{A}=0$ and $\theta^{C} \in(\lambda \bar{\theta}, \bar{\theta})$. Note that the optimal $\theta^{C}<\bar{\theta}$ for rewarding a challenger the whole patent rights, $\bar{\theta}$, generates no ex post benefits upon all other users, and in this case, $B_{j}$ 's search incentives will exceed the socially optimal level.

${ }^{23}$ When $\lambda=0$, $A$ 's payoff is $\left(1-\bar{\beta}_{0}^{s}\right) \bar{\theta} \Pi$ under current system, and may be improved to $\left(1-\bar{\beta}_{0}^{s}\right) \bar{\theta} \Pi+\bar{\beta}_{0}^{s} \theta^{C} S$. But, in a sense, this is because now $A$ can 'collude' and arrange a third party to get the prize and transfer the full prize $\theta^{C}$ to her, contradictory to the presumption that no side transfer of monopoly profit is possible.
} 
How to measure $\lambda$ : Previous analysis shows that the extent of collusion $\lambda$ is critical in determining the optimal challenge mechanism. We may evaluate this parameter by considering the underlying market structure. For instance, when a potential challenger competes with others in the product market, (partial) patent invalidation will intensify competition and hurt the challenger. The latter would have stronger incentives to collude. On the other hand, when there is spillover or positive network effects of technology adoption that cannot be appropriated via patent rights, a potential challenger may have less incentive to collude with the patent-holder in order to realize these benefits. ${ }^{24}$ Knowledge of market interactions helps determine the collusion parameter $\lambda$ and the construction of the optimal challenge policy.

Alternatively, the ex ante patent quality $\alpha$ may also have an impact in collusion incentives. In Appendix B, we present a sequential search case with two potential challengers. Suppose that a reward is given only to the first challenger. The second searcher will not search when the prior art has already been submitted. When observing that no patent challenge has occurred, on the other hand, the second searcher's decision will be based on the belief that the patent is a bogus one, which in turn is affected by the ex ante patent quality. Ceteris paribus, a lower patent quality means that it is more likely to face a bogus patent and thus more likely to find the information. Foreseeing the second searcher's efforts, the first searcher who discovers the information will be less willing to collude if the second searcher also finds the prior art and either shares the monopoly gain or submits the information and spoils the collusive deal. Higher search efforts from the second searcher, boosted by a lower patent quality, alleviates collusion in the first stage.

As a by-product, another interesting result in this case is that an optimal collusion may no require full collusion deterrence. Consider the collusion between the patentholder and the first challenger: If collusion is fully deterred, the second-searcher can only attribute no challenge to the search failure in the first round, which implies a lower probability of the existence of prior art. On the other hand, under a collusion accommodation policy, lack of a challenge doesn't convey this message and so preserves search incentives. This effect may cause the failure of the collusion-proofness principle.

Concluding remarks: To implement the challenge mechanism proposed in this paper will be no easy task. A proper reallocation of patent rights may require not only

\footnotetext{
${ }^{24}$ The two scenarios, we believe, correspond to the pharmaceutical and software industry, respectively.
} 
knowledge about collusion, but also a deep understanding of how patent rights are exploited in different industries. Above we have discussed the potential relationship between market competition and incentives to collude. Another channel through which the technology feature would affect the optimal mechanism is the various possible mappings from the technology space to product space. In this paper we've adopted a very simple construction in that aspect, and one could easily think of other situations. For instance, following the cumulative innovation literature (Green and Scotchmer, 1995), we may consider a setting where the challenger is himself a patent-holder, and so may face a 'counter-invalidation' threat should he dare to initiate a challenge procedure. Alternatively, we may also consider when the patent is not used 'offensively' (i.e., to extract licensing payment or exclude competitors), but 'defensively' as bargaining chips in licensing negotiations (Hall and Ziedonis, 2001). It would be useful and interesting to see how challenge incentives should be shaped by policy in these contexts.

\section{Appendix}

\section{A Proofs}

\section{Proposition 1}

Proof. In the collusion-free case, the optimal $\bar{\theta}=\theta^{*}$ and $\theta^{A}=0$. After a challenge, there is no point to reward the patent-holder $A$; and to encourage private search the patent authority can do better by raising $\theta^{C}$ instead of $\bar{\theta}$. For $\theta^{C}$, notice that the privately optimal search intensity $\beta^{s}$ satisfies $(1-\alpha)\left(\theta^{*} v^{e}+\theta^{C} S\right) \equiv c^{\prime}\left(\beta^{s}\right)$, with $\partial \beta^{s} / \partial \theta^{C}>0$. Social welfare is

$$
\begin{aligned}
& \mathcal{W}=\alpha\left(V^{e}-\theta^{*} D-K\right)+(1-\alpha)\left[\left(1-\beta^{s}\right)\left(V^{e}-\theta^{*} D\right)+\beta^{s}\left(V^{e}-\theta^{C}(N-1) d\right)\right]-c\left(\beta^{s}\right) \\
\Rightarrow & \frac{d \mathcal{W}}{d \theta^{C}}=(1-\alpha)\left(-\beta^{s}(N-1) d+\left\{\theta^{*}[(N-1) d-\pi]-\theta^{C}(N-1) v^{e}\right\} \frac{\partial \beta^{s}}{\partial \theta^{C}}\right) .
\end{aligned}
$$

The optimal $\theta^{C}>0$ if condition (2) holds at $\theta^{C}=0$.

Q.E.D.

\section{Lemma 1}

Proof. A general side contract specifies that (i) $B_{j}$ reports to $A$ his value $v_{j} \in\left\{v^{l}, v^{h}\right\}$ and promises not to challenge the patent; (ii) in return, $A$ transfers to $B_{j}$ a portion of patent rights $\tilde{\theta}_{-j}^{t}, t \in\{l, h\}$, again $B_{-j}$; and (iii) $B_{j}$ with value $v^{t}, t \in\{l, h\}$, can use 
the technology on his own production and generate a surplus $\left(1-\tilde{\theta}_{j}^{t}\right) v^{t}$, and possibly pays a fee $f^{t}$ to $A$. From the limited liability constraint and patent policy, $f^{t} \geq 0$ and $\tilde{\theta}_{-j}^{t} \leq \bar{\theta}$.

Let $A$ offer the side contract $\left\{\tilde{\theta}_{-j}^{t}, \tilde{\theta}_{j}^{t}, f^{t}\right\}_{t=h, l}$. The optimal side offer solves the following program (suppose both types of $B_{j}$ participate in the collusion)

$$
\begin{aligned}
(\mathcal{S}): \max \quad & \tilde{U}_{A}=\rho f^{h}+(1-\rho) f^{l}+\left[\bar{\theta}-\rho \tilde{\theta}_{-j}^{h}-(1-\rho) \tilde{\theta}_{-j}^{l}\right] S \\
\text { s.t. } \quad & (C I R)_{j}^{t}: \tilde{U}_{i}\left(v^{t}\right)=\left(1-\tilde{\theta}_{j}^{t}\right) v^{t}-f^{t}+\lambda \tilde{\theta}_{-j}^{t} S \geq v^{t}+\theta^{C} S, \quad t=h, l \\
& (I C)_{j}^{h}:\left(1-\tilde{\theta}_{j}^{h}\right) v^{h}-f^{h}+\lambda \tilde{\theta}_{-j}^{h} S \geq\left(1-\tilde{\theta}_{j}^{l}\right) v^{h}-f^{l}+\lambda \tilde{\theta}_{-j}^{l} S, \\
& (I C)_{j}^{l}:\left(1-\tilde{\theta}_{j}^{l}\right) v^{l}-f^{l}+\lambda \tilde{\theta}_{-j}^{l} S \geq\left(1-\tilde{\theta}_{j}^{h}\right) v^{l}-f^{h}+\lambda \tilde{\theta}_{-j}^{h} S .
\end{aligned}
$$

Assume $A$ colludes if and only if collusive payoff is strictly larger than outside option: $\tilde{U}_{A}>\theta^{A} S$. Define $\tau^{t} \equiv \lambda \tilde{\theta}_{-j}^{t} S-f^{t}$ as the net transfer from $A$ to $B$, when $v_{j}=v^{t}$, $t \in\{h, l\}$. In order to induce participation, $\tau^{t} \geq 0$. A's payoff can be written as

$$
\tilde{U}_{A}=\left\{\bar{\theta}-(1-\lambda)\left[\rho \tilde{\theta}_{-j}^{h}+(1-\rho) \tilde{\theta}_{-j}^{l}\right]\right\} S-\left[\rho \tau^{h}+(1-\rho) \tau^{l}\right] .
$$

From $\lambda \leq 1$, side transfer of patent rights entails loss to $A$. Therefore, given $\tau^{t} \geq 0$, if $f^{t}>0$ and so $\tilde{\theta}_{-j}^{t}>0$, the patent-holder's payoff can be increased by decreasing both $f^{t}$ and $\tilde{\theta}_{-j}^{t}$ while keeping $\tau^{t}$ unchanged. In an optimal collusive offer, $B_{j}$ pays no licensing fee, $f^{h}=f^{l}=0$.

Next, $(C I R)$ requires that $\left(\lambda \tilde{\theta}_{-j}^{t}-\theta^{C}\right) S \geq \tilde{\theta}_{j}^{t} v^{t} \geq 0$, for both $t$, and so $\lambda \tilde{\theta}_{-j}^{t} \geq \theta^{C}$. But then, when $\Delta \theta \leq 0, A$ 's payoff from collusion is

$$
\tilde{U}_{A}=\left[\bar{\theta}-\rho \tilde{\theta}_{-j}^{h}-(1-\rho) \tilde{\theta}_{-j}^{l}\right] S \leq\left(\bar{\theta}-\frac{\theta^{C}}{\lambda}\right) S \leq \theta^{A} S .
$$

Collusion doesn't take place in this case. On the other hand, if $\Delta \theta>0$, a feasible collusive offer is $\lambda \tilde{\theta}_{-j}^{t}=\theta^{C}$, and $\tilde{\theta}_{j}^{t}=0$, for both $t$. This offer gives the patent-holder a strictly higher payoff than no collusion. In fact, this is also the optimal side offer, for any $\tilde{\theta}_{j}^{t}>0$ necessarily increases $\tilde{\theta}_{-j}^{t}$ in order to satisfy $(C I R)_{j}^{t}$, which is costly to $A$. There is no benefit to screen $v_{j}$ in this case. $B_{j}$ gets a free license in a collusive offer. $A$ 's payoff at the optimal side offer is $\tilde{U}_{A}^{s}=\left(\bar{\theta}-\frac{\theta^{C}}{\lambda}\right) S$.

Finally, consider if $A$ screens $v_{j}$ by partial collusion, i.e. to collude only with one type of $B_{j}$. Consider a side offer $\left(\tilde{\theta}_{-j}, \tilde{\theta}_{j}\right)$ such that only one type's participation is satisfied (the same argument holds so that it is optimal to set $f=0$ ). First, it cannot be the case that only $B_{j}$ with high-value participates, for $v^{h}>v^{l}$ contradicts with partial collusion with high-value type, $\tilde{\theta}_{j} v^{l}>\left(\lambda \tilde{\theta}_{-j}-\theta^{C}\right) S-f \geq \tilde{\theta}_{j} v^{h}$. And second, to 
partially collude with the low-value type, it requires $\tilde{\theta}_{j} v^{h}>\left(\lambda \tilde{\theta}_{-j}-\theta^{C}\right) S-f \geq \tilde{\theta}_{j} v^{l}$, and so $\tilde{\theta}_{j}>0$ and $\lambda \tilde{\theta}_{-j}>\theta^{C}$. But from $\Delta \theta>0$ and $\lambda \tilde{\theta}_{-j}>\theta^{C}$, this is less profitable than colluding with both types, $\tilde{U}_{A}=\left[\rho \theta^{A}+(1-\rho)\left(\bar{\theta}-\tilde{\theta}_{-j}\right)\right] S<\tilde{U}_{A}^{s}$. $\quad$ Q.E.D.

\section{Lemma 2}

Proof. Because an optimal collusive offer contains only a side transfer $\tilde{\theta}_{-j} \geq 0$ and $A$ doesn't screen $B_{j}$ 's type, the patent authority can and should incorporate the optimal side contract into the patent policy in order to avoid the efficiency loss $(1-\lambda) \tilde{\theta}_{-j} S$. Notice that, due to a binding participation constraint at the collusion subgame, $B_{j}$ 's searching incentives are solely determined by the patent policy. Q.E.D.

\section{Proposition 2}

Proof. The optimal $\bar{\theta}=\theta^{*}$ if $N$ and $\alpha$ are large enough. For if not, when $n \geq 3$, we can decrease $\bar{\theta}$ by a small amount $\epsilon>0$ and increase $\theta^{C}$ by $\epsilon v^{e} / S<\epsilon$. The search intensity is unchanged, and when $\alpha>\frac{2}{N+2}$ the social welfare is increased by $\epsilon\left[\alpha D+(1-\alpha)\left(1-\beta^{s}\right) D-(1-\alpha) \beta^{s}(N-1) d \frac{v^{e}}{S}\right]>0$. Next, ignore $(I C)_{A}$, and denote the Lagrangian as $\mathcal{L}^{s}, F O C$ s are

$$
\begin{aligned}
& \frac{\partial \mathcal{L}^{s}}{\partial \theta^{A}}=-(1-\alpha) \beta^{s}(N-1) d+\mu \lambda \\
& \frac{\partial \mathcal{L}^{s}}{\partial \theta^{C}}=-(1-\alpha) \beta^{s}(N-1) d+\mu+(1-\alpha) \psi \frac{\partial \beta^{s}}{\partial \theta^{C}}
\end{aligned}
$$

where $\psi \equiv\left(\bar{\theta}-\theta^{A}-\theta^{C}\right)(N-1) d-\left[\bar{\theta}+\theta^{C}(N-1)\right] \pi$. The assumption that $c^{\prime \prime \prime} \geq 0$ ensures that $\partial^{2} \beta^{s} / \partial \theta^{A 2} \leq 0$ and thus the second-order condition will be satisfied.

Define the following terms: $\beta_{\lambda}^{s}$ by $(1-\alpha)\left(\theta^{*} v^{e}+\lambda \theta^{*} S\right) \equiv c^{\prime}\left(\beta_{\lambda}^{s}\right), \psi_{\lambda} \equiv\left(\theta^{*}-\lambda \theta^{*}\right)(N-$ $1) d-\left[\theta^{*}+\lambda \theta^{*}(N-1)\right] \pi, \phi_{\lambda} \equiv \beta_{\lambda}^{s}(N-1) d-\left.\psi_{\lambda} \frac{\partial \beta^{s}}{\partial \theta^{C}}\right|_{\beta_{\lambda}^{s}}$, and $\Lambda \equiv(1-\lambda) \phi_{\lambda}+\left.\psi_{\lambda} \frac{\partial \beta^{s}}{\partial \theta^{C}}\right|_{\beta_{\lambda}^{s}}$. Note that $\beta_{\lambda}^{s}$ is increasing in $\lambda$, while $\psi_{\lambda}$ is decreasing in $\lambda$, and so $\phi_{\lambda}$ increasing in $\lambda$. Also define $\underline{\lambda}$, $\hat{\lambda}$, and $\bar{\lambda}$ by $\phi(\underline{\lambda}) \equiv 0, \psi(\hat{\lambda}) \equiv 0$, and $\Lambda(\bar{\lambda}) \equiv 0$, respectively. The existence of $\bar{\lambda}$ is guaranteed by assuming $N$ large enough (more precisely, $N-1>$ $3 \pi^{m 2} / d^{2}$ ), so that $\Lambda$ is strictly decreasing in $\lambda$ over $\lambda \geq \hat{\lambda}$; and $\bar{\lambda}>\hat{\lambda}$. On the other hand, $\underline{\lambda}$ may not exist. When exists, it satisfies $\underline{\lambda}<\hat{\lambda}<\bar{\lambda}$.

With these definitions, when evaluating at $\theta^{C}=\lambda \theta^{*}$ and $\theta^{A}=0$, FOC s are

$$
\begin{aligned}
& \left.\frac{\partial \mathcal{L}^{s}}{\partial \theta^{A}}\right|_{\theta^{C}=\lambda \theta^{*}, \theta^{A}=0}=-(1-\alpha)\left(\phi_{\lambda}+\left.\psi_{\lambda} \frac{\partial \beta^{s}}{\partial \theta^{C}}\right|_{\beta_{\lambda}^{s}}\right)+\mu \lambda, \\
& \left.\frac{\partial \mathcal{L}^{s}}{\partial \theta^{C}}\right|_{\theta^{C}=\lambda \theta^{*}, \theta^{A}=0}=-(1-\alpha) \phi_{\lambda}+\mu .
\end{aligned}
$$


This policy is optimal if there exists $\mu \geq 0$ such that (7) is non-positive and (8) equals to zero. The three regimes are

(i) $\lambda \in[0, \underline{\lambda})$, if $\underline{\lambda}$ exists: In this case, $\phi_{\lambda}<0,(8)$ is strictly positive, the optimal $\theta^{C}>\lambda \theta^{*}$. This in turn implies that $(C P)$ slacks and so $\mu=0$. From (5), the optimal $\theta^{A}=0$.

(ii) $\lambda \in[\underline{\lambda}, \bar{\lambda}]$ : This regime can be further divided into two cases. For $\lambda \in[\underline{\lambda}, \hat{\lambda}]$, both $\phi_{\lambda}$ and $\psi_{\lambda}$ are non-negative. Let $\mu=(1-\alpha) \phi_{\lambda} \geq 0$ and plug it into (7), which then becomes non-positive. The policy $\theta^{C}=\lambda \theta^{*}$ and $\theta^{A}=0$ is optimal. Suppose $\lambda \in[\hat{\lambda}, \bar{\lambda}]$ and $\mu=(1-\alpha) \phi_{\lambda}$. Although $\psi_{\lambda} \leq 0$ in this range, by $\Lambda \geq 0,(7)$ is still non-positive. (iii) $\lambda \in(\bar{\lambda}, 1]$ : In this case, by $\Lambda<0$, there exists no $\mu \geq 0$ such that (7) is non-positive and (8) non-negative. And in any optimal $\mu$, condition (8) cannot be non-negative. For this leads to $\mu>0$, and again by $\Lambda<0,(7)$ is also positive. $(C P)$ slacks, contradictory to $\mu>0$. We then conclude that in optimum (7) must be strictly positive and (8) strictly negative. The optimal policy satisfies $\theta^{C}<\lambda \theta^{*}, \theta^{A}>0$, and $(C P)$ binding. (To further derive the optimal $\theta^{A}$, we can rewrite the program as a function of $\theta^{A}$ only. We omit it here.) Lastly, when $\lambda=1$, for any distribution of $\theta^{C}$ and $\theta^{A}$ that makes $(C P)$ binding, it must be $\psi<0$, and so condition (5) is strictly greater than (6). The optimal $\theta^{A}=\theta^{*}$ and $\theta^{C}=0 . \quad$ Q.E.D.
.

\section{B Collusion and Patent Quality under Sequen- tial Search}

When multiple players engage in sequential search, a lower patent quality may alleviate the collusion problem. It also provides an interesting case where the collusion-proof principle may fail. ${ }^{25}$

Suppose that two players $H_{1}$ and $H_{2}$ search sequentially. ${ }^{26}$ Let $H_{1}$ search first,

\footnotetext{
${ }^{25}$ Similar idea of using multiple auditors/supervisors to fight collusion can be found, among others, in Laffont and Martimort (1999) and Khalil and Lawarrée (2006). The latter study also endogenizes the collusion efficiency parameter $(\lambda)$ in a sequential supervision model, where tolerating early collusion is necessary to motivate the second supervision. However, in their auditing model, the second supervisor (an external auditor) is assumed not bribable and is appointed by the principal after the first supervisor (an internal auditor) sending the auditing report. The external auditor is a costly 'inspection device,' but the principal's lack of commitment to using this device. When collusion between the agent and the internal auditor is fully deterred, the principal loses incentives to hire the costly external auditor.

${ }^{26}$ We think this scenario captures some reality as it is difficult to imagine that all potential searchers
} 
and $H_{2}$ make a search decision after observing whether there is a patent challenge, but not whether $H_{1}$ has found the information or whether $A$ has colluded with $H_{1}$. For simplicity, assume that $H_{1}$ and $H_{2}$ are not technology users and both are bribable with $\lambda=1$. They also have the same two-point search technology: $H_{i}$ chooses search intensity $\beta_{i} \in\{0, \beta\}$, with $\beta \in(0,1)$, and cost $c(0)=0$ and $c(\beta)=c>0$. Given the existence of the prior art, the two's search result are independent of each other and cannot be observed by the other party.

Suppose $\theta^{A}=0$ (see the discussion below). The patent authority announces a policy $\left(\bar{\theta}, \theta^{C}\right)$, with the clause that $\theta^{C}$ is rewarded only to the first challenger. Since $H_{i}$ has no intrinsic interest in the technology, the challenge reward is worth $\theta^{C} \Pi$ to him. Let $A$ keep the whole bargaining power at the collusion subgame. This reward applies for both collusion and challenge paths.

$H_{1}$ will incur the cost $c$ if and only if $(1-\alpha) \beta \theta^{C} \Pi \geq c$, or, equivalently, $\theta^{C} \geq$ $\hat{\theta}_{1} \equiv \frac{1}{(1-\alpha) \beta} \frac{c}{\Pi}$. By the announced policy, $H_{2}$ won't search after $H_{1}$ 's challenge. If no challenge is observed, $H_{2}$ 's search decision hinges on the belief whether $H_{1}$ colludes with $A$, which is in turn affected by the patent authority's policy toward collusion. Denote $x$ as the probability that $H_{1}$ colludes with $A$. Observing no challenge from $H_{1}$, $H_{2}$ believes that the prior art exists with probability

$$
\frac{(1-\alpha)\left(1-\beta_{1}\right)+(1-\alpha) \beta_{1} x}{\alpha+(1-\alpha)\left(1-\beta_{1}\right)+(1-\alpha) \beta_{1} x}=\frac{(1-\alpha)\left[1-\beta_{1}(1-x)\right]}{1-(1-\alpha) \beta_{1}(1-x)}
$$

which is decreasing in $\alpha$ and increasing in $x$. $H_{2}$ searches, $\beta_{2}=\beta$, if and only if

$$
\frac{(1-\alpha)\left[1-\beta_{1}(1-x)\right]}{1-(1-\alpha) \beta_{1}(1-x)} \beta \theta^{C} \Pi \geq c \Rightarrow \theta^{C} \geq \hat{\theta}_{2}^{x} \equiv \frac{1}{(1-\alpha) \beta} \frac{1-(1-\alpha) \beta_{1}(1-x)}{1-\beta_{1}(1-x)} \frac{c}{\Pi} .
$$

Note that when $\beta_{1}=\beta, \hat{\theta}_{2}^{0}>\hat{\theta}_{2}^{1}=\hat{\theta}_{1}$. A policy that deters collusion between $H_{1}$ and $A$, $x=0$, raises the challenge reward needed to induce $H_{2}$ 's search. Under such a policy, $H_{2}$ rationally interprets no challenge as a result of $H_{1}$ 's search failure, which, given $\beta_{1}=\beta$, is in turn attributed to a higher $\alpha$, i.e. the patent is more likely to be valid. By contrast, if the policy allows collusion between $A$ and $H_{1}$, then lack of a challenge from $H_{1}$ does not send a bad news about the existence of prior art. Assume that $\bar{\theta}>\hat{\theta}_{1}$ and so, barring collusion, private search is useful to reduce ex post deadweight loss.

learn the granting of a patent at (roughly) the same time. Existing market competitors or professional 'bounty hunters' may observe closely the publication of patent issuance or applications. But new comers may acquire through time the technology skill or knowledge that could be used in patent challenges, not to mention professional researchers that learn about patent news from second-hand sources. 
Next, consider the policy toward collusion. We are mainly concerned with the case where $H_{1}$ finds the information. Note that deterring collusion in all events requires $\theta^{C}=\bar{\theta}$, since when $H_{1}$ fails we revert to the single-searcher case with $\lambda=1$. This policy also prevents collusion when $H_{1}$ finds the information, as $A$ cannot offer a higher bribe. Ignoring the event of the honest inventor, social welfare under this policy is $(1-\alpha)\left(V^{e}-\bar{\theta} D\right)-\left[1+\alpha+(1-\alpha)\left(1-\beta_{1}\right)\right] c$. By $x=0$ and $\bar{\theta}>\hat{\theta}_{1}$, both $H_{1}$ and $H_{2}$ exert search intensity $\beta$, but $H_{2}$ searches only when there is no challenge from $H_{1}$. In the current setting, the full collusion-deterrence policy is dominated by a case of no private challenge, $\theta^{C}<\hat{\theta}_{1}$, which leads to social welfare $(1-\alpha)\left(V^{e}-\bar{\theta} D\right) .{ }^{27}$

When $\theta^{C} \in\left(\hat{\theta}_{1}, \bar{\theta}\right)$, collusion occurs if $H_{2}$ alone finds the prior art. If $H_{1}$ finds and brings the information to $A$, their bargain is clouded by the possibility that $H_{2}$ finds the information as well. In that case, either $H_{2}$ challenges the patent and spoils the collusive deal between $H_{1}$ and $A$, or $H_{2}$ also colludes with $A$ and gets a share of $\bar{\theta}{ }^{28}$ Let $\beta_{2}^{x}$ be $H_{2}$ 's search intensity given collusion probability $x$. The following lemma describes the relationship between $\theta^{C}$ and collusion outcomes.

Lemma 3. (Partial collusion deterrence). Consider $\theta^{C}<\bar{\theta}$ and the subgame where $H_{1}$ has found the prior art.

When $\theta^{C}<\bar{\theta} / 2$, an equilibrium exists where $A$ colludes with both $H_{1}$ and $H_{2}$ (upon discovery); this is the unique equilibrium in the subgame when $0<\theta^{C}<\frac{1-\beta_{2}^{1}}{2-\beta_{2}^{1}} \bar{\theta}$.

When $\theta^{C} \geq \bar{\theta} / 2, A$ cannot collude with both searchers. And when $\theta^{C} \geq \frac{1-\beta_{2}^{1}}{2-\beta_{2}^{1}} \bar{\theta}$, an equilibrium exists where there is no collusion between $A$ and $H_{2}$. In addition, (i) if $\theta^{C}<\left(1-\beta_{2}^{1}\right) \bar{\theta}, A$ colludes with $H_{1}$ with probability one; and (ii) if $\theta^{C} \geq\left(1-\beta_{2}^{0}\right) \bar{\theta}$, there is no collusion between $H_{1}$ and $A$.

Proof. A bribe of $\theta^{C}$ is necessary to collude with $H_{2}$. For $H_{1}$, if he expects no challenge from $H_{2}$, then he will accept $A$ 's side offer $\theta^{C}$. Therefore when $\theta^{C}<\bar{\theta} / 2, A$ can collude with both searcher whatever $H_{1}$ 's expectation. There is always an full collusionaccommodation equilibrium. Even if $H_{1}$ expects $H_{2}$ to search and challenge the patent, the maximal bribe he'll ask is $\theta^{C} /\left(1-\beta_{2}^{x}\right)$, where $\beta_{2}^{x}$ is $H_{2}$ 's search intensity given collusion probability $x$ between $H_{1}$ and $A$. As long as $\theta^{C} /\left(1-\beta_{2}^{x}\right)+\theta^{C}<\bar{\theta}$, or $\theta^{C}<\frac{1-\beta_{2}^{x}}{2-\beta_{2}^{x}} \bar{\theta}<\bar{\theta} / 2, A$ can collude with both searchers.

${ }^{27}$ This hinges on the assumptions that $\lambda=1$ and, again, that patent challenges provide no ex ante benefits.

${ }^{28}$ The assumption of an enforceable side contract prevents $H_{1}$ from running to the patent authority after accepting a side offer. For $A$, since she is protected by limited liability and $\theta^{A}=0$, when $H_{2}$ challenges the patent, $A$ cannot pay the promised bribe. 
Suppose that $\theta^{C} \geq \frac{1-\beta_{2}^{1}}{2-\beta_{2}^{1}} \bar{\theta}$ and $H_{1}$ demands a large bribe $\theta^{C} /\left(1-\beta_{2}^{x}\right)$, expecting $H_{2}$ 's challenge probability $\beta_{2}^{x}$. Since $A$ 's maximal willingness to pay is $\bar{\theta}$, (i) if $\theta^{C} /\left(1-\beta_{2}^{1}\right)<$ $\bar{\theta}$, there is collusion between $H_{1}$ and $A$, and $H_{2}$ sets his search intensity at $\beta_{2}^{1}$; and (ii) if $\theta^{C} /\left(1-\beta_{2}^{0}\right) \geq \bar{\theta}$, there is no collusion between $H_{1}$ and $A$, and $H_{2}$ sets his search intensity at $\beta_{2}^{0}$.

Note that renegotiation is ignored. Once the side deal is reached, $H_{1}$ will insist on the payment $A$ has promised. This can be justified by assuming that only $A$, but not $H_{1}$, observes whether $H_{2}$ has found the information or not. This implies that $H_{1}$ receives no new information after signing the side contract, and so his outside option remains the same in renegotiation. If $H_{1}$ agrees a lower payment after $H_{2}$ finds the information, then $A$ will send this request even when $H_{2}$ actually fails. $A$ could simply arrange someone else to pretend to be a searcher. This opportunistic behavior would $\begin{array}{ll}\text { handicap renegotiation. } & \text { Q.E.D. }\end{array}$

When $\frac{1-\beta_{2}^{1}}{2-\beta_{2}^{1}} \bar{\theta} \leq \theta^{C} \leq \bar{\theta} / 2$ and so there are multiple equilibria in the collusion subgame, we will focus on the partial deterrence equilibrium. ${ }^{29}$ Also, the fact that $\theta^{C}<\bar{\theta}$ but $H_{2}$ challenges the patent implies collusion between $A$ and $H_{1}$. However, the patent authority cannot use this inference to punish $A$ or $H_{1}$ because of limited liability protection. In addition, the patent authority may not know the identity of potential challengers unless they submit the information.

Due to a two-point search technology $\beta_{2} \in\{0, \beta\}$, it is necessary to induce $H_{2}$ 's search in order to deter collusion between $H_{1}$ and $A$ while keeping $\theta^{C}<\bar{\theta}$. The challenge reward should then satisfy $\theta^{C} \geq \max \left\{(1-\beta) \bar{\theta}, \hat{\theta}_{2}^{0}\right\}$. This gives us an expression of collusion-proof constraint in the form of $\theta^{C} \geq(1-\hat{\lambda}) \bar{\theta}$, where $\hat{\lambda}$ is either equal to $\beta$ or determined by $\hat{\theta}_{2}^{0}$. When $\hat{\theta}_{2}^{0}>(1-\beta) \bar{\theta}$, which is more likely to hold when $\beta$ increases, a lower patent quality reduces the prize required to deter collusion between $H_{1}$ and $A$, for $\hat{\theta}_{2}^{0}$ increasing in $\alpha$. Lower patent quality may alleviate the collusion problem.

Proposition 4. (Collusion and patent quality). Consider sequential search and the event when $H_{1}$ has found the information. A lower patent quality may alleviate the collusion problem between $H_{1}$ and $A$ : The lower bound of necessary reward to induce $x=0$, $\max \left\{(1-\alpha) \bar{\theta}, \hat{\theta}_{2}^{0}\right\}$, is weakly increasing in $\alpha$.

\footnotetext{
${ }^{29}$ The collusion accommodation equilibrium could be eliminated by introducing a non-bribable type of $H_{2}$. When there is small but positive probability that $H_{2}$ will challenge the patent, $H_{1}$ will demand a higher bribe than $\theta^{C}$.
} 
To evaluate welfare effect for $\theta^{C} \in\left(\hat{\theta}_{1}, \bar{\theta}\right)$, set $\theta^{C}=\theta^{C, 0}=\max \left\{(1-\beta) \bar{\theta}, \hat{\theta}_{2}^{0}\right\}$, and so social welfare of deterring collusion between $H_{1}$ and $A$ is $\left.\mathcal{W}\right|_{x=0}=(1-\alpha)\left[\beta\left(V^{e}-\theta^{C, 0} D\right)+(1-\beta)\left(V^{e}-\bar{\theta} D\right)\right]-[1+\alpha+(1-\alpha)(1-\beta)] c$.

$\theta^{C, 0}$ applies if and only if $H_{1}$ has found and submitted the information; $H_{2}$ then will not search. But if $A$ and $H_{1}$ is allowed to collude, $x=1$, by Lemma 3, the patent authority only needs to set $\theta^{C, 1}=\max \left\{\hat{\theta}_{1}, \frac{1-\beta}{2-\beta} \bar{\theta}\right\}$. By the same lemma, this policy is feasible when $\hat{\theta}_{1}<(1-\beta) \bar{\theta}$. ${ }^{30}$ Social welfare under this policy is

$$
\left.\mathcal{W}\right|_{x=1}=(1-\alpha)\left[\beta^{2}\left(V^{e}-\theta^{C, 1} D\right)+\left(1-\beta^{2}\right)\left(V^{e}-\bar{\theta} D\right)\right]-2 c .
$$

The policy $\theta^{C, 1}$ applies when $H_{1}$ and $H_{2}$ find the information, for $A$ cannot collude with both. $H_{2}$ will incur $c$ for sure because $H_{1}$ will not challenge the patent.

Compare the two policies:

$$
\begin{aligned}
\left.\mathcal{W}\right|_{x=1}-\left.\mathcal{W}\right|_{x=0} & =(1-\alpha)\left[\beta \theta^{C, 0}+(1-\beta) \bar{\theta}-\beta^{2} \theta^{C, 1}-\left(1-\beta^{2}\right) \bar{\theta}\right] D-(1-\alpha) \beta c \\
& =(1-\alpha) \beta\left\{\left[\theta^{C, 0}-\beta \theta^{C, 1}-(1-\beta) \bar{\theta}\right] D-c\right\}
\end{aligned}
$$

Relative to preventing $A$ from colluding with $H_{1}$, partial collusion accommodation improves social welfare by requiring a lower challenge reward $\theta^{C, 1} \leq \theta^{C, 0}$, at a cost of more frequent search from $H_{2}$, who also searches when $H_{1}$ has found the information but colludes with $A$. The welfare difference, expression (9), can't be positive if $\theta^{C, 0}=$ $(1-\beta) \bar{\theta} \geq \hat{\theta}_{2}^{0}$. On the other hand, we can have $\left.\mathcal{W}\right|_{x=1}>\mathcal{W}_{x=0}$ whether $\hat{\theta}_{1} \gtrless \frac{1-\beta}{2-\beta} \bar{\theta}$. The following proposition gives specific conditions if $\bar{\theta}=\theta^{*}$. As can be shown, $x=0$ is more likely to be optimal when $\beta$ is larger. ${ }^{31}$

Proposition 5. (Failure of collusion-proofness principle). The collusion-proofness principle may not hold in the sequential search case. It is optimal to accommodate collusion between $H_{1}$ and $A,\left.\mathcal{W}\right|_{x=1}>\left.\mathcal{W}\right|_{x=0}$, which is possible only if $\hat{\theta}_{2}^{0}>(1-\beta) \bar{\theta}$, i.e., $\theta^{C, 0}=\hat{\theta}_{2}^{0}$.

\footnotetext{
${ }^{30}$ When $x=1, \hat{\theta}_{2}^{1}=\hat{\theta}_{1}$ and so $\beta_{2}=\beta$ if $H_{1}$ searches.

${ }^{31}$ For completeness, we also compare the partial-accommodation policy $\theta^{C, 1}$ with the full-accommodation policy $\theta^{C}=0$ :

$\left.\mathcal{W}\right|_{x=1}-(1-\alpha)\left(V^{e}-\bar{\theta} D\right)=(1-\alpha) \beta^{2}\left(\bar{\theta}-\theta^{C, 1}\right) D-2 c= \begin{cases}(1-\alpha) \frac{\beta^{2}}{2-\beta} \frac{d}{\pi} K-2 c & \text { if } \hat{\theta}_{1}<\frac{1-\beta}{2-\beta} \bar{\theta} \\ (1-\alpha) \beta^{2} \frac{d}{\pi} K-\left(2+\beta \frac{d}{\pi}\right) c & \text { if } \hat{\theta}_{1}>\frac{1-\beta}{2-\beta} \bar{\theta}\end{cases}$
}

at $\bar{\theta}=\theta^{*}$. Again, both are more likely to be positive when $\beta$ is larger. 
Furthermore, if $\bar{\theta}=\theta^{*}$, then condition (9) is strictly positive if:

(i) for $\hat{\theta}_{1}>\frac{1-\beta}{2-\beta} \bar{\theta}$ and so $\theta^{C, 1}=\hat{\theta}_{1}$,

$$
\left[\frac{(1-\beta)^{2}+\alpha \beta}{(1-\alpha) \beta(1-\beta)} \frac{d}{\pi}-1\right] c>(1-\beta) \frac{d}{\pi} K
$$

subject to $\max \left\{\frac{(1-\alpha) \beta(1-\beta)}{2-\beta} K, \frac{(1-\alpha) \beta(1-\beta)^{2}}{1-\beta(1-\alpha)} K\right\}<c<(1-\alpha) \beta(1-\beta) K$; and

(ii) for $\theta^{C, 1}=\frac{1-\beta}{2-\beta} \bar{\theta}>\hat{\theta_{1}}$,

$$
\left[\frac{1-\beta(1-\alpha)}{(1-\alpha) \beta(1-\beta)} \frac{d}{\pi}-1\right] c>\frac{2(1-\beta)}{2-\beta} \frac{d}{\pi} K,
$$

subject to $\frac{(1-\alpha) \beta(1-\beta)^{2}}{1-\beta(1-\alpha)} K<c<\frac{(1-\alpha) \beta(1-\beta)}{2-\beta} K$.

Proof. Case (i) requires $\frac{1-\beta}{2-\beta} \bar{\theta}<\hat{\theta}_{1}<(1-\beta) \bar{\theta}<\hat{\theta}_{2}^{0}<\bar{\theta}$, and case (ii) requires $\hat{\theta}_{1}<\frac{1-\beta}{2-\beta} \bar{\theta}<(1-\beta) \bar{\theta}<\hat{\theta}_{2}^{0}<\bar{\theta}$. We can obtain these results by inserting $\bar{\theta}=$ $\theta^{*}=K / \Pi, \theta^{C, 0}=\hat{\theta}_{2}^{0}=\frac{1-\beta(1-\alpha)}{1-\beta} \hat{\theta}_{1}$, and $\hat{\theta}_{1}=\frac{1}{(1-\alpha) \beta} \frac{c}{\Pi}$ into condition (9) and these requirements.

Q.E.D.

Remark 1. (Optimality of $\theta^{A}=0$ ). Previous analysis shows that $\theta^{A}>0$ only to dilute search incentives. With a binary search decision, a higher reward won't induce more search once the threshold $\hat{\theta}_{i}$ is passed. Also note that a strictly positive $\theta^{A}$ facilitates collusion, for $A$ can use this to pay the bribe to $H_{1}$ even after $H_{2}$ challenges the patent. $\|$

Remark 2. (Rationally ignorant patent office). The positive relationship between patent quality and the extent of collusion has an interesting implication for the role of the patent office in the patent-granting process, which has been abstracted in this paper. While the patent quality can be improved with more resources injected in the patent office and better performance by patent examiners, it comes at a cost of aggravating post-issuance collusion. This may provide another support of Lemley (2006)'s 'rationally ignorant' patent office. 


\section{Ex Post Analysis of the Single-User Case (Not for publication)}

In this appendix, we consider the special case of $N=1 .^{32}$ Suppose that the unique user of patented technology $B$ values the technology at $v \in\left\{v^{h}, v^{l}\right\}$ and has the search ability. The patent protection thus is exercised against $B$. To slightly abuse the notation, a patent policy consists of two elements $\Theta=\left(\bar{\theta}, \theta^{A}\right)$ : $A$ is granted $\bar{\theta} \geq \theta^{*}$ when there is no patent challenge, and $\theta^{A}$ otherwise. Intuitively, the optimal policy should satisfy $\bar{\theta} \geq \theta^{A} .{ }^{33}$ Collusion is not an issue here; the patent authority needs only to deal with inadequate private incentives to search.

An interesting result in this special case is that any policy satisfying $\bar{\theta}>\theta^{A}$ leads to over-search, including the ex post efficient policy $\Theta^{*} \equiv\left(\theta^{*}, 0\right)$, where the inventor's innovation incentive is satisfied and the patent is entirely invalidated after prior art submission. The see the discrepancy between private and social incentives to search, let's keep the timing that $B$ chooses the search intensity $\beta$ before learning $v$. Given a policy $\Theta=\left(\bar{\theta}, \theta^{A}\right), B$ 's challenge benefit is the production surplus over the narrowed patent right $\bar{\theta}-\theta^{A}$. The privately optimal search intensity $\hat{\beta}$ is determined by

$$
\hat{\beta}=\arg \max _{\beta}(1-\alpha) \beta\left(\bar{\theta}-\theta^{A}\right) v^{e}-c(\beta) \Rightarrow F O C:(1-\alpha)\left(\bar{\theta}-\theta^{A}\right) v^{e} \equiv c^{\prime}(\hat{\beta}) .
$$

On the other hand, society benefits from the reduction of deadweight loss associated with monopoly pricing. The socially optimal search intensity $\beta^{*}$ is determined by

$$
\beta^{*}=\arg \max _{\beta}(1-\alpha) \beta\left(\bar{\theta}-\theta^{A}\right) d-c(\beta) \Rightarrow F O C:(1-\alpha)\left(\bar{\theta}-\theta^{A}\right) d \equiv c^{\prime}\left(\beta^{*}\right) .
$$

Since $v^{e}>d$, private incentives to search are greater than social incentives; there is over-search, $\hat{\beta}>\beta^{*}$.

Patent policy: Taking search incentives into account, the ex post optimal policy solves

$$
\begin{aligned}
\max _{\bar{\theta}, \theta^{A}} & \alpha\left(v^{e}-\bar{\theta} d-K\right)+(1-\alpha)\left[v^{e}-\hat{\beta} \theta^{A} d-(1-\hat{\beta}) \bar{\theta} d\right]-c(\hat{\beta}) \\
\text { s.t. } & \bar{\theta} \geq \theta^{*} \text { and } c^{\prime}(\hat{\beta}) \equiv(1-\alpha)\left(\bar{\theta}-\theta^{A}\right) v^{e}
\end{aligned}
$$

${ }^{32}$ To motivate this case, consider a patented product. Patent-holder $A$ lacks commercialization capacity and must license patent rights to an incumbent $B$. There may be potential entrants, but assume that a positive entry fee and fierce competition (e.g. Bertrand competition) drive post-entry profit to zero. Alternatively, there may be barriers to entry other than IPRs. When $B$ acts as the sole manufacturer, the private information $v$ may be a market demand or production cost parameter.

${ }^{33}$ Otherwise it is $A$, not $B$, who would search in order to find the information and expand patent protection. 


\begin{tabular}{|c|c|c|c|c|c|c|}
\hline $\begin{array}{l}0 \\
\square\end{array}$ & $\begin{array}{l}1 \\
1\end{array}$ & $\begin{array}{c}1.5 \\
\square\end{array}$ & 2 & 2.5 & 3 & 4 \\
\hline policy & $\begin{array}{l}\text { R\&D and } \\
\text { patenting }\end{array}$ & $\begin{array}{l}\text { uninformed } \\
\text { search }\end{array}$ & $v$ & $\begin{array}{l}\text { informed } \\
\text { search }\end{array}$ & $\begin{array}{l}\text { challenge } \\
\text { upon discovery }\end{array}$ & licensing \\
\hline
\end{tabular}

Figure 3: Timing with informed search

The optimal $\bar{\theta}=\theta^{*}$ : Raising patent protection under no challenge above $\theta^{*}$ only creates more deadweight loss and induces higher search intensity, which already exceeds the socially optimal level. More interestingly, the optimal $\theta^{A}$ may be greater than zero, because doing so would discourage private search. The optimal $\theta^{A}>0$ if and only if $\hat{\beta}$ is sufficiently sensitive to the policy adjustment. The following proposition provides the exact condition.

Proposition 6. When the challenger is the unique technology user, private incentives cause over-search. The optimal $\bar{\theta}=\theta^{*}$. It is optimal to set $\theta^{A}>0$ if and only if

$$
\left.\frac{\left(\bar{\theta}-\theta^{A}\right)}{\hat{\beta}} \frac{\partial \hat{\beta}}{\partial\left(\bar{\theta}-\theta^{A}\right)}\right|_{\Theta^{*}}>\frac{d}{\pi} .
$$

Proof. The optimality of $\bar{\theta}=\theta^{*}$ is trivial. Condition (12) comes from a positive firstorder condition when evaluated at $\Theta^{*}$ :

$$
-(1-\alpha) \hat{\beta}\left(\Theta^{*}\right) d-\left.(1-\alpha) \pi \theta^{*} \frac{\partial \hat{\beta}}{\partial \theta^{A}}\right|_{\Theta^{*}}=-(1-\alpha) \hat{\beta}\left(\Theta^{*}\right) d\left(1+\left.\frac{\pi}{d} \frac{\theta^{*}}{\hat{\beta}} \frac{\partial \hat{\beta}}{\partial \theta^{A}}\right|_{\Theta^{*}}\right) .
$$

Q.E.D.

Signaling effect of private search: Alternatively, the signaling effect of a patent challenge may discourage private search. Consider a situation where $B$ learns the true value $v$ before engaging in information search. Referring to Figure 1, we shall call this scenario "informed search" (search at time 2.5) and the case we considered in the main text "uninformed search" (search at time 1.5). Under informed search, different values $v^{t}$ may lead to different intensities $\beta^{t}, t \in\{h, l\}$. Even if $A$ cannot 
observe the search intensity, ${ }^{34}$ as long as $\beta^{h} \neq \beta^{l}$, an opportunistic inventor acquires new information about $v$ from the search outcome. ${ }^{35}$

For instance, when $\beta^{l}<<\beta^{h} \simeq 1$, the opportunistic patent-holder infers no challenge and so search failure from low search intensity due to low value $v^{l}$, i.e., the lack of a challenge signifies low value; she accordingly lowers the licensing fee to $v^{l}$. The user with high value $v^{h}$, then, has an incentive to lower his search intensity in order to capitalize on this signaling effect and realize surplus $v^{h}-v^{l}$.

Define $\bar{\rho} \equiv \frac{v^{l}}{v^{h}}$. A's optimal pricing strategy is $v^{h}\left(v^{l}\right)$ when she holds belief $\operatorname{Pr}\left(v^{h}\right) \geq$ $\bar{\rho}\left(\operatorname{Pr}\left(v^{h}\right)<\bar{\rho}\right.$, respectively). Given $\Theta$, define $\hat{\beta}^{t}, t \in\{h, l\}$, such that

$$
(1-\alpha)\left(\bar{\theta}-\theta^{A}\right) v^{t} \equiv c^{\prime}\left(\hat{\beta}^{t}\right) .
$$

$\hat{\beta}^{t}$ is the privately optimal search level under the licensing fee $v^{h}$. The following lemma gives the shape of equilibria in this game (see the proof for the full characterization).

Lemma 4. Consider the single-user, informed search case. When A observes only the search outcome, but not the intensity, there is no equilibrium in which both types of user $B$ choose the same intensity. And

$\mathcal{E}^{1}$ : In the unique pure strategy equilibrium, if it exists, $B$ with value $v^{t}$ chooses equilibrium intensity $\hat{\beta}^{t}, t=h, l$, and $A$ always offers a licensing fee $v^{h}$. This equilibrium exists if and only if

$$
\frac{\rho\left(1-\hat{\beta}^{h}\right)}{\rho\left(1-\hat{\beta}^{h}\right)+(1-\rho)\left(1-\hat{\beta}^{l}\right)} \geq \bar{\rho} ;
$$

$\mathcal{E}^{2}$ : If condition (14) fails, an equilibrium exists where

- $B$ with $v^{l}$ still chooses $\hat{\beta}^{l}$; and $B$ with $v^{h}$ lowers his search intensity to some level in the open interval $\left(\hat{\beta}^{l}, \hat{\beta}^{h}\right)$;

- the honest $A$ always offers a licensing fee $v^{h}$. The opportunistic A offers a licensing fee $v^{h}$ if there is a patent challenge; otherwise, she randomizes between the two offers $v^{h}$ and $v^{l}$, with a belief $\bar{\rho}$.

Proof. A low-value type user always gets zero surplus over $\bar{\theta}$ and $\theta^{A}$, whatever the patent-holder's pricing strategy. His equilibrium search intensity $\hat{\beta}^{l}$ is determined

\footnotetext{
${ }^{34}$ This may not always be the case. Suppose, for instance, that $B$ has no ability to search, but can post a bounty on BountyQuest's website (see Section 1). $B$ then influences the search intensity via the amount of bounty. By design this amount is public information.

${ }^{35}$ There is no such learning for the honest inventor, since no prior art can be found whatever the intensity.
} 
according to (13). Given $\left(\beta^{h}, \hat{\beta}^{l}\right)$, denote the updated belief as $\rho_{\emptyset}\left(\rho_{p}\right)$ when nothing is found (the prior art is found, respectively), with

$$
\rho_{\emptyset}=\frac{\rho\left(1-\beta^{h}\right)}{\rho\left(1-\beta^{h}\right)+(1-\rho)\left(1-\hat{\beta}^{l}\right)} \text { and } \rho_{p}=\frac{\rho \beta^{h}}{\rho \beta^{h}+(1-\rho) \hat{\beta}^{l}} \text {. }
$$

Consider first the pure strategy equilibrium.

$\diamond$ Suppose $\beta^{h}=\hat{\beta}^{l}$ in equilibrium, then search result reveals no new information, $\rho_{\emptyset}=\rho_{p}=\rho$. The opportunistic inventor charges $v^{h}$ whether there is challenge or not. The high-value type user gets

$$
\begin{aligned}
& \pi_{B}^{h}=\alpha(1-\bar{\theta}) v^{h}+(1-\alpha)\left[\beta^{h}\left(1-\theta^{A}\right) v^{h}+\left(1-\beta^{h}\right)(1-\bar{\theta}) v^{h}\right]-c\left(\beta^{h}\right) \\
\Rightarrow \quad & \left.\frac{\partial \pi_{B}^{h}}{\partial \beta^{h}}\right|_{\beta^{h}=\hat{\beta}^{l}}=(1-\alpha)\left(\bar{\theta}-\theta^{A}\right) v^{h}-c^{\prime}\left(\hat{\beta}^{l}\right)>0 .
\end{aligned}
$$

He will deviate to a higher level of search intensity; this can't be an equilibrium.

$\diamond$ For an equilibrium with different intensities, first note that $\hat{\beta}^{h}>\hat{\beta}^{l}$ in any equilibrium; otherwise, $\rho_{\emptyset}>\rho \geq \bar{\rho}$ along the equilibrium path, while $\rho_{p} \gtrless \bar{\rho}$. Monopoly price remains at $v^{h}$ if no challenge. When there is a challenge, the lower bound of the highvalue user's surplus is $\left(1-\theta^{A}\right) v^{h}$. But then the equilibrium $\hat{\beta}^{h}>\hat{\beta}^{l}$, a contradiction, for the minimal equilibrium intensity solves

$$
\max _{\beta^{h}}(1-\alpha)\left\{\beta^{h}\left[\theta^{A} \cdot 0+\left(1-\theta^{A}\right) v^{h}\right]+\left(1-\beta^{h}\right)(1-\bar{\theta}) v^{h}\right\}-c\left(\beta^{h}\right) .
$$

From $\hat{\beta}^{h}>\hat{\beta}^{l}$, along the equilibrium path, beliefs are such that $\rho_{p}>\rho>\rho_{\emptyset}$ and $\rho_{p}>\bar{\rho}$. The optimal pricing when the prior art is found is $v^{h}$. Depending on $\rho_{\emptyset} \gtrless \bar{\rho}$ :

(i) if $\rho_{\emptyset}<\bar{\rho}$, the opportunistic inventor sets the licensing fee at $v^{l}$ over $\theta^{A}$, but then the high-value user's optimal search intensity solves

$$
\begin{aligned}
& \quad \max _{\beta^{h}}(1-\alpha)\left\{\beta^{h}\left(1-\theta^{A}\right) v^{h}+\left(1-\beta^{h}\right)\left[\bar{\theta} \Delta v+(1-\bar{\theta}) v^{h}\right]\right\}-c\left(\beta^{h}\right) \\
& \Rightarrow \quad F O C:(1-\alpha)\left[\left(\bar{\theta}-\theta^{A}\right) v^{h}-\bar{\theta} \Delta v\right] \equiv c^{\prime}\left(\hat{\beta}^{h}\right) .
\end{aligned}
$$

This leads to a contradiction as $\left(\bar{\theta}-\theta^{A}\right) v^{h}-\bar{\theta} \Delta v \leq\left(\bar{\theta}-\theta^{A}\right) v^{l}$, where $\Delta v \equiv v^{h}-v^{l}$.

(ii) if $\rho_{\emptyset} \geq \bar{\rho}$, the opportunistic inventor sets the licensing fee at $v^{h}$ over $\theta^{A}$, the optimal search intensity is determined by $(1-\alpha)\left(\bar{\theta}-\theta^{A}\right) v^{h} \equiv c^{\prime}\left(\hat{\beta}^{h}\right)$. For this to be an equilibrium, $\hat{\beta}^{h}$ cannot be too large. The necessary and sufficient condition of existence is $\rho_{\emptyset}\left(\hat{\beta}^{t}\right) \geq \bar{\rho}$, i.e. condition (14).

$\diamond$ If condition (14) fails, there is no pure equilibrium. An equilibrium exists such that the honest inventor always offers a licensing fee $v^{h}$; the opportunistic inventor offers a 
licensing fee $v^{h}$ in the case of patent challenge, and she randomizes the price by offering $v^{l}$ with probability $y \in(0,1)$ when no challenge; and the low-value user always chooses $\hat{\beta}^{l}$ while the high-value user chooses $\beta_{y}^{h} \in\left(\hat{\beta}^{l}, \hat{\beta}^{h}\right)$. The equilibrium $y$ and $\beta_{y}^{h}$ satisfy

$$
\frac{\rho\left(1-\beta_{y}^{h}\right)}{\rho\left(1-\beta_{y}^{h}\right)+(1-\rho)\left(1-\hat{\beta}^{l}\right)}=\bar{\rho} \text { and }(1-\alpha)\left[\left(\bar{\theta}-\theta^{A}\right) v^{h}-y \bar{\theta} \Delta v\right] \equiv c^{\prime}\left(\beta_{y}^{h}\right) \text {. }
$$

The existence and uniqueness of $\beta_{y}^{h} \in\left(\hat{\beta}^{l}, \hat{\beta}^{h}\right)$ is guaranteed by the failure of condition (14). Once $\beta_{y}^{h}$ is fixed, a unique $y \in(0,1)$ can be found.

Q.E.D.

In $\mathcal{E}^{2}$, the user learns perfectly that $A$ is opportunistic by observing the price offer $v^{l}$. For simplicity, we assume away any search opportunity after this learning and the elicitation of this information by the patent authority. ${ }^{36}$

In the pure strategy equilibrium $\mathcal{E}^{1}$, the licensing fee is fixed at $v^{h}$. Prior art submission narrows patent protection from $\bar{\theta}$ to $\theta^{A}$, but social welfare increases only when $v=v^{l}$. Put differently, given this pricing strategy and search intensity, when the value of the technology increases from $v^{l}$ to $v^{h}$, private search benefit is raised from $(1-\alpha) \beta\left(\bar{\theta}-\theta^{A}\right) v^{l}$ to $(1-\alpha) \beta\left(\bar{\theta}-\theta^{A}\right) v^{h}$, but social benefit is reduced from $(1-\alpha) \beta\left(\bar{\theta}-\theta^{A}\right) v^{l}$ to zero. An increase in the technology value causes the privately and socially optimal search intensity to adjust along opposite directions.

Equilibrium $\mathcal{E}^{2}$ exists when condition (14) fails. This is the case when $\hat{\beta}^{h}$ is large, i.e. a high-value user searches so intensively that, in case of search failure, the opportunistic inventor suspects that she most likely faces a low-value user and therefore reduces the licensing fee. The 'no challenge' signal, then, confers benefit on the high-value user and thus justifies lower search intensity. ${ }^{37}$

Relative to $\mathcal{E}^{1}$, private behavior in equilibrium $\mathcal{E}^{2}$ is more 'social friendly' because the high-value user reduces his search intensity (although still exceeds the social optimal level), and the opportunistic inventor lowers the licensing fee with some probability.

Proposition 7. (Signaling). The private behavior in equilibrium $\mathcal{E}^{2}$ is more in line with social interests in terms of reduced search and the probability of a lower licensing fee.

\footnotetext{
${ }^{36}$ Otherwise, the opportunistic inventor would prefer to mimic the honest type and refrain from offering $v^{l}$. This is socially costly.

${ }^{37}$ In this equilibrium, the high-value user still submits after discovery, for $\left(1-\theta^{A}\right) v^{h}>y\left(v^{h}-v^{l}\right)+(1-\bar{\theta}) v^{h}$, where $y$ is the probability that the opportunistic inventor offers $v^{l}$ when there is no challenge.
} 
Remark. (BountyQuest revisited). Besides the standard free rider argument, the signaling effect described here may help explain the failure of BountyQuest (Section 1). ${ }^{38}$ Nevertheless, Proposition 7 also suggests that this failure could be welfare-enhancing in some cases.

Remark. (Signaling from the patent-holder). Signaling may also appear on the patentholder side. If we alter the timing of the game so that the licensing stage comes before the search stage, an opportunistic patent-holder may be tempted to charge a lower fee in order to reduce search intensity. But since no honest inventor would do this, this offer reveals the patent-holder's true type and may backfire by restoring search incentives.

\section{Simultaneous search and the collusion-proof mechanism (Not for publication)}

This appendix considers how the optimal mechanism characterized in Proposition 2 should be modified if two users search simultaneously. Assume that the two usersearchers make the search decision simultaneously with a common cost $c(\cdot)$. For simplicity, let the search results be realized independently, and the number of users be large enough. To be comparable with section 3, we assume that when one searcher has found the prior art, he observes whether the other has also found it. The only friction in collusion therefore is the loss in transfer $1-\lambda .^{39}$

For simplicity, keep the policy restriction to exempt a user from licensing obligation when he submits the prior art. A policy is still a triplet $\left(\bar{\theta}, \theta^{A}, \theta^{C}\right)$, but now $\theta^{C}$ is assumed shared equally if both searchers submit the information. ${ }^{40}$ The collusionproofness principle holds, with the same $(C P)$, although the collusion gains reduces to a proportion of $(N-2) \pi$ when both have found the information. We first look for

\footnotetext{
${ }^{38}$ According to the company rule, the identity of both bounty posters and hunters are kept secret and submitted information is disclosed only to the poster. This should reduce free riding.

${ }^{39}$ Otherwise, if only $A$ observes how many users have found the information, she has incentives to claim that both have found it in order to reduce the collusive offer when, in fact, only one has found the information. Knowing this, the searcher won't accept such an offer and so collusion may breakdown even when there are gains from doing so.

${ }^{40}$ We use a "no arbitrage" argument to justify the implicit assumption that the patent rights allocation after challenge cannot depend on the number of challengers. Suppose, say, $\theta^{C}$ is higher when there are two challengers. If only one has found the prior art, the unique searcher can reveal the information to any third party and realize the higher payoff. By the same toke, $\theta^{A}$ is independent of the number of challengers.
} 
the symmetric equilibrium in search intensity, denoted as $\beta^{m}$. Given the other chooses $\beta^{m}$, a searcher's optimal intensity is determined by

$$
\begin{aligned}
\max _{\tilde{\beta}}(1-\alpha)\left\{\beta^{m} \tilde{\beta}\left[v^{e}+\frac{\theta^{C}}{2}(N-2) \pi\right]+\left(1-\beta^{m}\right) \tilde{\beta}\left(v^{e}+\theta^{C} S\right)\right. \\
\left.+\beta^{m}(1-\tilde{\beta})\left(1-\theta^{C}\right) v^{e}+\left(1-\beta^{m}\right)(1-\tilde{\beta})(1-\bar{\theta}) v^{e}\right\}-c(\tilde{\beta}) \\
\quad c^{\prime}\left(\beta^{m}\right) \equiv(1-\alpha)\left\{v^{e}\left[\bar{\theta}-\beta^{m}\left(\bar{\theta}-\theta^{C}\right)\right]+\theta^{C}\left(S-\beta^{m} \frac{\Pi}{2}\right)\right\} .
\end{aligned}
$$

It is easy to see that, under the same policy, $\beta^{m}<\beta^{s}$. The presence of the other searcher reduces one's search incentives because of the sharing of the prize when both have found the information and free-riding, i.e. when $\theta^{C}<\bar{\theta}$, one can profit from a lower patent power due to the other's search. The policy's incentive effect to boost search is also weaker. When measured at the same policy and $\beta$,

$$
\frac{\partial \beta^{s}}{\partial \theta^{C}}=\frac{(1-\alpha) S}{c^{\prime \prime}}>\frac{(1-\alpha)}{c^{\prime \prime}}\left[S-\beta\left(\frac{\Pi}{2}-v^{e}\right)\right]>\frac{\partial \beta^{m}}{\partial \theta^{C}} .
$$

The patent authority's optimization program is

$$
\begin{aligned}
(\mathcal{P})^{m}: \max _{\bar{\theta}, \theta^{A}, \theta^{C}} & \mathcal{W}^{m}=\alpha\left(V^{e}-\bar{\theta} D-K\right)+(1-\alpha)\left[V^{e}-\left(1-\beta^{m}\right)^{2} \bar{\theta} D\right. \\
& \left.-2 \beta^{m}\left(1-\beta^{m}\right)\left(\theta^{A}+\theta^{C}\right)(N-1) d-\beta^{m 2}\left(\theta^{A}+\theta^{C}\right)(N-2) d\right]-2 c\left(\beta^{m}\right) \\
\text { s.t. } \quad(I C)_{A}: \bar{\theta} \geq \theta^{*}, \quad(C P): \Delta \theta \leq 0, & (I C)_{c}: c^{\prime}\left(\beta^{m}\right) \equiv(1-\alpha)\left\{v^{e}\left[\bar{\theta}-\beta^{m}\left(\bar{\theta}-\theta^{C}\right)\right]+\theta^{C}\left(S-\beta^{m} \frac{\Pi}{2}\right)\right\} .
\end{aligned}
$$

Besides the difference in search intensity, multiple-search also slightly reduces the social loss of the challenge reward when both have found the information to $(N-2) d$. The optimal policy still takes the shape of three regimes: only when suboptimal private incentives to search become a serious concern will the optimal policy depart from the lest costly way to deter collusion, $\theta^{C}=\lambda \theta^{*} .{ }^{41}$ But the ranges of the three regimes may be affected. For instance, consider a very small $\lambda$ so that collusion is not the dominant concern. Although free riding lowers private incentives to search, and so the patent authority is more willing to give additional reward, a smaller incentive effect of policy $\left(\partial \beta^{m} / \partial \theta^{C}<\partial \beta^{s} / \partial \theta^{C}\right)$ also makes it more costly to restore search intensity. This trade-off may expand or shrink regime (i), relative to the previous case. Similar reasoning holds at the high end of $\lambda$, where duplication of search cost worsens the over-search problem.

\footnotetext{
${ }^{41}$ Large $N$ and $\alpha$ guarantee the optimality of $\bar{\theta}=\theta^{*}$ for similar argument in the proof of Proposition 2.
} 
As an example, suppose $c(\beta)=C \beta^{2} / 2$ and $N \rightarrow \infty$. $^{42}$ The next proposition shows that, relative to the single search case, in simultaneously multiple-search regime (i) shrinks while regime (iii) may or may not expand.

Proposition 8. Suppose $c(\beta)=C \beta^{2} / 2$ and $N \rightarrow \infty$. When there are two searchers $\left(\right.$ program $\left.(\mathcal{P})^{m}\right)$, relative to the case where only one users searches (program $\left.(\mathcal{P})^{s}\right)$, regime (i) shrinks; and regime (iii) expands when $\pi^{2} \leq 3 d^{2}$, or when $\pi^{2}>3 d^{2}$ but $\pi$ and $C$ are not too large.

Proof. Suppose $c(\beta)=\frac{C}{2} \beta^{2}$, with $C>0$. When evaluated at $\bar{\theta}=\theta^{*}$, optimal private search intensities and the policy $\theta^{C}$ 's incentive effects are

$$
\begin{aligned}
& \beta^{s}=\frac{1-\alpha}{C}\left(\theta^{*} v^{e}+\theta^{C} S\right)>\beta^{m}=\frac{C}{C+\Delta} \beta^{s}, \text { and } \\
& \frac{\partial \beta^{s}}{\partial \theta^{C}}=\frac{(1-\alpha) S}{C}>\frac{\partial \beta^{m}}{\partial \theta^{C}}=\frac{C}{C+\Delta} \frac{\partial \beta^{s}}{\partial \theta^{C}}-\frac{C}{(C+\Delta)^{2}} \beta^{s} \frac{\partial \Delta}{\partial \theta^{C}}>0,
\end{aligned}
$$

where $\Delta=(1-\alpha)\left[\left(\theta^{*}-\theta^{C}\right) v^{e}+\theta^{C} \frac{\Pi}{2}\right]$. To consider the limiting case $N \rightarrow \infty$, we let $C$ be proportional to $N$ in order to constrain $\beta^{s}$ below one. Solving $\underline{\lambda}$ and $\bar{\lambda}$ in the proof of Proposition 2, as $N \rightarrow \infty$,

$$
\underline{\lambda}=\frac{N \pi d-(\pi+d)^{2}}{(N-1) \pi(\pi+2 d)} \rightarrow \frac{d}{\pi+2 d} \text { and } \bar{\lambda} \rightarrow \frac{2 d}{\pi+2 d}
$$

for $\bar{\lambda}$ is the positive root of

$$
\lambda^{2}(\pi+2 d) \pi(N-1)-\lambda\left[2 \pi d(N-1)-\pi^{2}-(\pi+d) d\right]-(\pi+d) d=0 .
$$

Since a reward of $\theta^{C}=\lambda \theta^{*}$ stops at $\bar{\lambda}$, to ensure $\beta^{s}<1$ when $N \rightarrow \infty$, we require

$$
\beta^{s}=\frac{(1-\alpha) \theta^{*}}{C}\left[v^{e}+\bar{\lambda} S\right]<1 \Rightarrow C>(1-\alpha) \theta^{*} \frac{(\pi+2 d) \pi}{2 d} N .
$$

(i). To show that regime (i) shrinks, denote the Lagrangian of program $(\mathcal{P})^{m}$ as $\mathcal{L}^{m}$, and the multiplier of $(C P)$ as $\mu$. When evaluating at $\theta^{A}=0$ and $\theta^{C}=\lambda \bar{\theta}=\lambda \theta^{*}$, the FOC with respect to $\theta^{C}$ is

$$
\begin{aligned}
\frac{\partial \mathcal{L}^{m}}{\partial \theta^{C}}= & -(1-\alpha)\left\{2\left[\beta^{m}(N-1) d-\psi_{\lambda} \frac{\partial \beta^{m}}{\partial \theta^{C}}\right]-\beta^{m 2} D\right. \\
& \left.+2 \theta^{*} \beta^{m}\left[(N-1) d-\pi-\lambda(N-2)\left(d+\frac{\pi}{2}\right)\right] \frac{\partial \beta^{m}}{\partial \theta^{C}}\right\}+\mu \\
= & -(1-\alpha)\left\{\frac{2 C}{C+\Delta}\left[\beta^{s}(N-1) d-\psi_{\lambda} \frac{\partial \beta^{s}}{\partial \theta^{C}}\right]+\frac{2 C \beta^{s} \psi_{\lambda}}{(C+\Delta)^{2}} \frac{\partial \Delta}{\partial \theta^{C}}-\left(\frac{C}{C+\Delta} \beta^{s}\right)^{2} D\right. \\
& \left.+2 \theta^{*}\left(\frac{C}{C+\Delta}\right)^{2} \beta^{s}\left[(N-1) d-\pi-\lambda(N-2)\left(d+\frac{\pi}{2}\right)\right]\left[\frac{\partial \beta^{s}}{\partial \theta^{C}}-\frac{\beta^{s}}{C+\Delta} \frac{\partial \Delta}{\partial \theta^{C}}\right]\right\}+\mu,
\end{aligned}
$$

\footnotetext{
${ }^{42}$ To keep $\beta^{s}<1$, we let $C$ be proportional to $N$. See the proof.
} 
where $\psi_{\lambda}$ is as defined in the proof of Proposition 2. At $\underline{\lambda}$, by definition, $\beta^{s}(N-1) d \equiv$ $\psi_{\lambda}\left(\partial \beta^{s} / \partial \theta^{C}\right)$. The sign of $\frac{\partial \mathcal{L}^{m}}{\partial \theta^{C}}$ is determined by

$$
\begin{aligned}
& -\frac{2 C \beta^{s} \psi_{\lambda}}{(C+\Delta)^{2}} \frac{\partial \Delta}{\partial \theta^{C}}+\left(\frac{C}{C+\Delta} \beta^{s}\right)^{2} D \\
& -2 \theta^{*}\left(\frac{C}{C+\Delta}\right)^{2} \beta^{s}\left[(N-1) d-\pi-\lambda(N-2)\left(d+\frac{\pi}{2}\right)\right]\left[\frac{\partial \beta^{s}}{\partial \theta^{C}}-\frac{\beta^{s}}{C+\Delta} \frac{\partial \Delta}{\partial \theta^{C}}\right] \\
= & \frac{C \beta^{s}}{(C+\Delta)^{2}}\left\{-2 \psi_{\lambda} \frac{\partial \Delta}{\partial \theta^{C}}+C \beta^{s} D-2 \theta^{*} C\left[(N-1) d-\pi-\lambda(N-2)\left(d+\frac{\pi}{2}\right)\right]\left[\frac{\partial \beta^{s}}{\partial \theta^{C}}-\frac{\beta^{s}}{C+\Delta} \frac{\partial \Delta}{\partial \theta^{C}}\right]\right\} .
\end{aligned}
$$

As $N \rightarrow \infty$,

$$
\begin{aligned}
& (N-1) d-\pi-\underline{\lambda}(N-2)\left(d+\frac{\pi}{2}\right) \rightarrow \frac{N}{2} d-\pi, \quad \Delta \rightarrow \frac{(1-\alpha) \theta^{*}}{\pi+2 d}\left[(\pi+d)^{2}+\pi d \frac{N}{2}\right], \\
& 2 \psi_{\lambda} \frac{\partial \Delta}{\partial \theta^{C}}=\frac{\beta^{s}(N-1) d}{\partial \beta^{s} / \partial \theta^{C}}(1-\alpha)\left(\Pi-v^{e}\right) \rightarrow(1-\alpha) \theta^{*}(\pi+2 d)(\pi+d)\left(N-\frac{v^{e}}{\pi}\right), \\
& C \beta^{s} D \rightarrow(1-\alpha) \theta^{*}\left[v^{e}+\frac{d}{\pi+2 d}(N-1) \pi\right] N d, \quad \text { and } \\
& 2 \theta^{*} C\left[(N-1) d-\pi-\lambda(N-2)\left(d+\frac{\pi}{2}\right)\right]\left[\frac{\partial \beta^{s}}{\partial \theta^{C}}-\frac{\beta^{s}}{C+\Delta} \frac{\partial \Delta}{\partial \theta^{C}}\right] \\
& \rightarrow(1-\alpha) \theta^{*}(N d-2 \pi)\left\{(N-1) \pi-\left(\frac{N \pi}{2}-v^{e}\right) \frac{(1-\alpha) \theta^{*}\left[(\pi+2 d) v^{e}+(N-1) \pi d\right]}{(\pi+2 d) C+(1-\alpha) \theta^{*}\left[(\pi+d)^{2}+\frac{N}{2} \pi d\right]}\right\} .
\end{aligned}
$$

For $N$ sufficiently large, the comparison is dominated by $N^{2}$. Collecting relevant terms (and ignore $\left.(1-\alpha) \theta^{*}\right)$,

$$
\begin{aligned}
& \frac{\pi d^{2}}{\pi+2 d} N(N-1)-N d\left\{(N-1) \pi-\frac{N}{2} \pi \frac{(1-\alpha) \theta^{*}\left[(\pi+2 d) v^{e}+(N-1) \pi d\right]}{(\pi+2 d) C+(1-\alpha) \theta^{*}\left[(\pi+d)^{2}+\frac{N}{2} \pi d\right]}\right\} \\
<\quad & N \pi d\left\{-\frac{\pi+d}{\pi+2 d}(N-1)+\frac{N}{2} \frac{(\pi+2 d) v^{e}+(N-1) \pi d}{\frac{(\pi+2 d)^{2} \pi}{2 d} N+(\pi+d)^{2}+\frac{\pi d}{2} N}\right\} \\
\rightarrow \quad & N \pi d\left[-\frac{\pi+d}{\pi+2 d}(N-1)+\frac{d^{2}}{(\pi+2 d)^{2}+d^{2}} N\right]<0 .
\end{aligned}
$$

Therefore, for $N$ large enough, regime (i) shrinks when there are two searchers.

(ii). To show that regime (iii) may expand, by the same token, at $\bar{\lambda}$, by $\partial \mathcal{L}^{m} / \partial \theta^{C}=0$, the FOC with respect to $\theta^{A}$ is

$$
\begin{aligned}
\frac{\partial \mathcal{L}^{m}}{\partial \theta^{A}}= & -(1-\alpha)\left\{2\left[(1-\lambda) \beta^{m}(N-1) d+\lambda \psi_{\lambda} \frac{\partial \beta^{m}}{\partial \theta^{C}}\right]\right. \\
& \left.-(1-\lambda) \beta^{m 2} D-2 \lambda \theta^{*} \beta^{m}\left[(N-1) d-\pi-\lambda(N-2)\left(d+\frac{\pi}{2}\right)\right] \frac{\partial \beta^{m}}{\partial \theta^{C}}\right\} \\
= & -(1-\alpha)\left\{\frac{2 C}{C+\Delta}\left[(1-\lambda) \beta^{s}(N-1) d+\lambda \psi_{\lambda} \frac{\partial \beta^{s}}{\partial \theta^{C}}\right]-\lambda \psi_{\lambda} \frac{2 C \beta^{s}}{(C+\Delta)^{2}} \frac{\partial \Delta}{\partial \theta^{C}}-(1-\lambda)\left(\frac{C \beta^{s}}{C+\Delta}\right)^{2} D\right. \\
& \left.-2 \lambda \theta^{*}\left(\frac{C}{C+\Delta}\right)^{2} \beta^{s}\left[(N-1) d-\pi-\lambda(N-2)\left(d+\frac{\pi}{2}\right)\right]\left[\frac{\partial \beta^{s}}{\partial \theta^{C}}-\frac{\beta^{s}}{C+\Delta} \frac{\partial \Delta}{\partial \theta^{C}}\right]\right\},
\end{aligned}
$$


where $(1-\lambda) \beta^{s}(N-1) d \equiv-\lambda \psi_{\lambda}\left(\partial \beta^{s} / \partial \theta^{C}\right)$ at $\bar{\lambda}$. When evaluated at $\bar{\lambda}$ and $\theta^{C}=\bar{\lambda} \theta^{*}$, the sign of FOC is determined by

$$
\begin{aligned}
& 2 \lambda \psi_{\lambda} \frac{\partial \Delta}{\partial \theta^{C}}+(1-\lambda) C \beta^{s} D+2 \lambda \theta^{*} C\left[(N-1) d-\pi-\lambda(N-2)\left(d+\frac{\pi}{2}\right)\right]\left[\frac{\partial \beta^{s}}{\partial \theta^{C}}-\frac{\beta^{s}}{C+\Delta} \frac{\partial \Delta}{\partial \theta^{C}}\right] \\
= & (1-\alpha) \lambda \psi_{\lambda}\left(N \pi-v^{e}\right)+(1-\lambda) C \beta^{s} N d+2 \lambda C\left[\psi_{\lambda}+\lambda \theta^{*}\left(d+\frac{N}{2} \pi\right)\right]\left[\frac{\partial \beta^{s}}{\partial \theta^{C}}-\frac{\beta^{s}}{C+\Delta} \frac{\partial \Delta}{\partial \theta^{C}}\right] .
\end{aligned}
$$

Since at $\bar{\lambda}$, as $N \rightarrow \infty$,

$$
\begin{aligned}
& \bar{\lambda} \psi_{\bar{\lambda}}=-(1-\bar{\lambda}) \frac{\beta^{s}(N-1) d}{\partial \beta^{s} / \partial \theta^{C}}=-(1-\bar{\lambda}) \theta^{*}\left(v^{e}+\bar{\lambda} S\right) \frac{d}{\pi} \rightarrow-\frac{d \theta^{*}}{\pi+2 d}\left(v^{e}+\frac{2 d}{\pi+2 d} S\right), \\
& \bar{\lambda} \psi_{\bar{\lambda}}+\bar{\lambda}^{2} \theta^{*}\left(d+\frac{N}{2} \pi\right) \rightarrow-\frac{d(\pi-d)}{\pi+2 d} \theta^{*}, \text { and } \Delta \rightarrow \frac{(1-\alpha) \theta^{*} \pi}{\pi+2 d}\left(v^{e}+d N\right),
\end{aligned}
$$

for $N$ sufficiently large the sign of FOC is determined by

$$
\begin{aligned}
& (1-\alpha) \theta^{*} \frac{d v^{e}}{\pi+2 d}\left(v^{e}+\frac{2 d}{\pi+2 d} S\right)-(1-\alpha) \theta^{*} \frac{2 d(\pi-d)}{\pi+2 d}\left[S-\frac{(1-\alpha) \theta^{*}\left(v^{e}+\frac{2 d}{\pi+2 d} S\right)}{C+\frac{(1-\alpha) \theta^{*} \pi}{\pi+2 d}\left(v^{e}+N d\right)}\left(\frac{\Pi}{2}-v^{e}\right)\right] \\
= & \frac{(1-\alpha) \theta^{*} d}{\pi+2 d}\left\{v^{e}+2 S\left[\frac{d v^{e}}{\pi+2 d}-(\pi-d)\right]+(\pi-d)\left(N \pi-2 v^{e}\right) \frac{(1-\alpha) \theta^{*}\left(v^{e}+\frac{2 d}{\pi+2 d} S\right)}{C+\frac{(1-\alpha) \theta^{*} \pi}{\pi+2 d}\left(v^{e}+N d\right)}\right\} .
\end{aligned}
$$

If $\frac{d v^{e}}{\pi+2 d} \geq \pi-d$, or if $3 d^{2} \geq \pi^{2}, \frac{\partial \mathcal{L}^{m}}{\partial \theta^{A}}$ is strictly positive, and so regime (iii) expands. Even if $\pi^{2}>3 d^{2}$, considering the terms of $N$, for $N$ sufficiently large, FOC is strictly positive whenever

$$
\begin{aligned}
& -\frac{2\left(\pi^{2}-3 d^{2}\right)}{\pi+2 d} S+(\pi-d) \frac{(1-\alpha) \theta^{*}\left(v^{e}+\frac{2 d}{\pi+2 d} S\right)}{C+\frac{(1-\alpha) \theta^{*} \pi}{\pi+2 d}\left(v^{e}+N d\right)} N \pi>0 \\
\Rightarrow \quad & C<(1-\alpha) \theta^{*} \frac{\pi d^{2}(\pi+d)}{(\pi+2 d)\left(\pi^{2}-3 d^{2}\right)} N .
\end{aligned}
$$

As long as $\frac{\pi+2 d}{2 d}<\frac{d^{2}(\pi+d)}{(\pi+2 d)\left(\pi^{2}-3 d^{2}\right)}$, or, $\pi^{4}+4 \pi^{3} d+\pi^{2} d^{2}-14 \pi d^{3}-14 d^{4}<0$, there exists $C$ such that regime (iii) expands when there are two searchers. Otherwise, regime (iii) shrinks.

Q.E.D.

\section{E An Evaluation of the Bounty System (Not for publication)}

Two straightforward questions with any bounties are: What is the correct reward, and where does the money come from? Intuitively, the amount of bounty should reflect the social benefit of patent challenges. This benefit is correlated with the value of 
the patented technology, and the private sector would be expected to have a better knowledge of it. A classical problem, then, is how to elicit this information. Given the information asymmetry between technology users and the patent-holder, the former, especially of the high-value type, may be reluctant to report truthfully the value of the technology if there is a significant probability of search or challenge failure. Even when they don't need to pay the bounty, revealing his true type may hurt the high-value user by raising the licensing fee in case of no challenge. For the second question, we discuss in turn whether the bounty is paid by private parties or the patent authority. In our model, there are two candidates of private parties, technology users $B_{i}$ and the patent-holder $A$.

Bounty paid by technology users: Given the ex post participation constraint, i.e., the option of not using the technology, to require technology users to pay the bounty is equivalent to granting patent rights to the successful challenger. The same collusion problem emerges if the bounty is not large enough. If the amount of bounty is set by the patent authority, we end up with a compulsory licensing scheme, a policy the court is reluctant to adopt. ${ }^{43}$

\section{Private Bounty Paid by the Patent-holder: If the bounty is paid by} the patent-holder $A$ (Thomas, 2001), this serves as a punishment to opportunistic patenting. The quality of issued patents will improve since the incentives to apply for a bogus patent are lower. A risk of this punishment is to dampen R\&D incentives if there is some probability of 'type II' error, i.e. a good patent (when $k=K$ ) is struck down. ${ }^{44}$ To incorporate pecuniary punishment, let's relax the limited liability constraint. ${ }^{45}$ An argument that a private bounty may not be optimal, then, serves to justify this constraint.

For simplicity, we consider the single-user case under uninformed search. ${ }^{46}$ To

\footnotetext{
${ }^{43}$ And for a complete evaluation, compulsory licensing should also be applied to the original patent-holder.

${ }^{44}$ Among others, this error may come from the patent authority's improper treatment of non-obviousness criterion. For instance, hindsight bias may give rise to the wrong perception that, after realization, an invention is easier to achieve than it actually was.

${ }^{45}$ This may not always be feasible. For instance, even large firms will spin off smaller entities to market the patent licenses if they have to pay damages. This will make them 'judgement-proof,' i.e. the spin-offs have shallow pockets and won't pay damages.

${ }^{46}$ In the multi-user case, this bounty by itself cannot deter collusion, as it is merely a zero-sum transfer between the two colluding parties. The gains from collusion, i.e. the rent extracted from other users, are not affected. But a bounty changes the threat point at the collusion bargaining, and reduces the return from
} 
introduce type II error, suppose for any $\beta$, there is a probability $\varepsilon \beta \in(0, \beta)$ to find the prior art when $k=K$. When finding the information, the updated probability that $k=0$ is

$$
\frac{(1-\alpha) \beta}{\alpha \varepsilon \beta+(1-\alpha) \beta}=\frac{(1-\alpha)}{\alpha \varepsilon+(1-\alpha)} .
$$

Type II error thus dilutes the information value of prior art. But we keep $\varepsilon$ small enough so that the prior art remains an informative signal about $k=0$, and assume the patent authority invalidates the patent after submission, $\theta^{A}=0 .{ }^{47} \mathrm{~A}$ policy consists of two elements: The patent protection $\bar{\theta}$ when there is no challenge, and the bounty $b \geq 0$ paid by $A$ when the prior art is submitted. Given a pair $(\bar{\theta}, b)$, expected profits are $U_{A}^{h}=(1-\varepsilon \beta) \bar{\theta} \pi-K-\varepsilon \beta b$ for an honest inventor, and $U_{A}^{o}=(1-\beta) \bar{\theta} \pi-\beta b$ for an opportunistic inventor. $B$ 's payoff is $U_{B}=\alpha\left[(1-\bar{\theta}) v^{e}+\varepsilon \beta\left(b+\bar{\theta} v^{e}\right)\right]+(1-\alpha)(1-z) v^{e}+$ $(1-\alpha) z\left[(1-\bar{\theta}) v^{e}+\beta\left(b+\bar{\theta} v^{e}\right)\right]-c(\beta)$, where $z$ is the probability that an opportunistic inventor applies for a patent. The innovation incentive constraint is $U_{A}^{h} \geq 0$. When it is satisfied, social welfare is

$$
\mathcal{W}^{I I}=\alpha\left[v^{e}-(1-\varepsilon \beta) \bar{\theta} d-K\right]+(1-\alpha)(1-z) v^{e}+(1-\alpha) z\left[v^{e}-(1-\beta) \bar{\theta} d\right]-c(\beta)
$$

The optimal policy exhibits a 'bang-bang' property: Either there is no deterrence $(z=1$ and $b=0)$, or opportunistic patenting is fully deterred $(z=0)$. The latter, however, may not be feasible.

Lemma 5. (Bang-bang property). Suppose $\varepsilon>0$. The optimal policy is either no bounty, $b=0$ and $U_{A}^{o}>0$, or a deterrence bounty, $b>0$ and $U_{A}^{h}=U_{A}^{o}=z=0$.

Proof. For the no-deterrence regime: When $\varepsilon$ and $\beta>0$, for any $b \geq 0$, to satisfy $U_{A}^{h} \geq 0$ we must set $\bar{\theta}>\theta^{*}$. If at $b>0, U_{A}^{o}>0$ and so the opportunistic inventor applies for a patent for sure, a small reduction of $b$ doesn't alter this behavior. But this change decreases $\bar{\theta}$ and search intensity $\beta$. Both increase welfare under single-user case. Next, consider the deterrence regime, $U_{A}^{h} \geq 0 \geq U_{A}^{o}$.

$\diamond$ If $U_{A}^{o}<0$, then $z=0$. If we keep $\bar{\theta}$ the same but slightly decrease $b$ while maintaining $U_{A}^{o}<0$, then $\beta$ is reduced and $U_{A}^{h}$ increases. This reduction in $b$ is feasible and welfareenhancing.

opportunistic patenting. This will improve the patent quality and alleviate the collusion problem because of its rarer occurrence.

${ }^{47}$ There is no point to punish the inventor by $b$ and reward her with $\theta^{A}$ at the same time. 
$\diamond$ If $U_{A}^{h}>0$. From previous result,

$$
U_{A}^{o}=0 \Rightarrow \bar{\theta} \pi=\frac{\beta b}{1+\beta} \Rightarrow U_{A}^{h}=\frac{1-\varepsilon}{1-\beta} \beta b-K>0 .
$$

Reduce $\bar{\theta}$ by a small amount $\delta_{\theta}<0$ while keeping $b$ the same. To keep $U_{A}^{o}=0$, the new equilibrium must have a lower $\beta$. Denote the change as $\delta_{\beta}<0$. For this to be true,

$$
(1-\alpha)\left(b+\bar{\theta} v^{e}\right) \delta_{z}+[\alpha \varepsilon+(1-\alpha) z] v^{e} \delta_{\theta}<0 \Rightarrow \delta_{z}<-\frac{[\alpha \varepsilon+(1-\alpha) z] v^{e} \delta_{\theta}}{(1-\alpha)\left(b+\bar{\theta} v^{e}\right)},
$$

where $\delta_{z} \gtrless 0$ is the change of $z$. Social welfare is changed by

$$
\begin{aligned}
\delta_{\mathcal{W}} & =-d[\alpha(1-\varepsilon \beta)+(1-\alpha) z(1-\beta)] \delta_{\theta}-(1-\alpha)(1-\beta) \bar{\theta} d \delta_{z}-[\alpha \varepsilon+(1-\alpha) z] b \delta_{\beta} \\
& >-d \delta_{\theta}\left\{\alpha(1-\varepsilon \beta)+(1-\alpha) z(1-\beta)-\frac{[\alpha \varepsilon+(1-\alpha) z](1-\beta) v^{e} \bar{\theta}}{b+\bar{\theta} v^{e}}\right\}-[\alpha \varepsilon+(1-\alpha) z] b \delta_{\beta} .
\end{aligned}
$$

It can be seen that $\delta_{\mathcal{W}}>0$. As long as the change is small enough, and so $\delta_{\beta}$ not too large, we can keep $U_{A}^{h}>0$. This change is feasible.

For the optimality of $z=0$, from $U_{A}^{h}=U_{A}^{o}=0$, in equilibrium the patent protection required $\bar{\theta}^{d}$ is solely determined by $K, \pi$, and $\varepsilon$, and the search intensity $\beta^{d}$ is decreasing in $b$. Since an opportunistic inventor is indifferent between applying for a patent or not, and a low $\beta$ increases social welfare, it is optimal to select $z=0$ Q.E.D.

At the no-deterrence regime, a bounty $b>0$ has no direct welfare effect, but it increases search intensity $\beta$ and the necessary patent protection $\bar{\theta}$ to encourage innovation. Both decrease social welfare. On the other hand, if the patent authority wants to deter opportunistic patenting, at the optimal policy there is full deterrence, $z=0$, and the bounty is set at no more than necessary, i.e. $U_{A}^{o}=0$, since raising $b$ entails a cost of an increase in $\bar{\theta}$.

We first claim that when $\varepsilon>0$, the deterrence bounty may not be feasible. To show this, note that the condition $U_{A}^{o} \leq 0$ imposes a lower bound on $b: \beta b \geq(1-\beta) \bar{\theta} \pi$, which then implies an upper bound on $U_{A}^{h}: U_{A}^{h} \leq(1-\varepsilon \beta) \bar{\theta} \pi-K-\varepsilon(1-\beta) \bar{\theta} \pi=(1-\varepsilon) \bar{\theta} \pi-K$. But if

$$
(1-\varepsilon) \pi<K \leq\left(1-\varepsilon \beta_{0}\right) \pi
$$

where $\beta_{0}$ is the search intensity when $b=0$, there is no feasible patent policy $\bar{\theta} \leq 1$ to implement such a bounty. Condition (15) holds for a high type II error $\varepsilon$, or a large $K$ 
so that an honest inventor already needs a very strong patent protection $\bar{\theta}$ absent any punishment.

Second, we show that the deterrence bounty may be too costly. Suppose $K \leq$ $(1-\varepsilon) \pi$. From Lemma 5 , in any deterrence equilibrium $U_{A}^{h}=U_{A}^{o}=0$, then ${ }^{48}$

$\bar{\theta}^{d}=\frac{K}{(1-\varepsilon) \pi}, \quad \beta^{d}=\frac{K}{K+(1-\varepsilon) b^{d}}, \quad$ where $\alpha \varepsilon\left(b^{d}+v^{e} \frac{K}{(1-\varepsilon) \pi}\right) \equiv c^{\prime}\left(\frac{K}{K+(1-\varepsilon) b^{d}}\right)$.

While in the no-deterrence regime, the optimal policy is

$$
\bar{\theta}^{n}=\frac{K}{\left(1-\varepsilon \beta^{n}\right) \pi}, \quad \text { where }[\alpha \varepsilon+(1-\alpha)] \frac{K}{\left(1-\varepsilon \beta^{n}\right) \pi} v^{e} \equiv c^{\prime}\left(\beta^{n}\right) .
$$

We have $\bar{\theta}^{n}<\bar{\theta}^{d}$ and $\beta^{n} \gtrless \beta^{d}$. The difference of social welfare is

$\mathcal{W}^{I I}\left(\bar{\theta}^{d}, \beta^{d}\right)-\mathcal{W}^{I I}\left(\bar{\theta}^{n}, \beta^{n}\right)=(1-\alpha)\left(1-\beta^{n}\right) \bar{\theta} d+\alpha\left[\left(\bar{\theta}^{n}-\bar{\theta}^{d}\right)-\varepsilon\left(\beta^{n} \bar{\theta}^{n}-\beta^{d} \bar{\theta}^{d}\right)\right] d-\left[c\left(\beta^{d}\right)-c\left(\beta^{n}\right)\right]$.

Suppose both the cost difference and $\varepsilon$ are not large enough to dominate the comparison. Relative to no deterrence, the deterrence policy increases social welfare by thwarting opportunistic patenting that cannot be detected by $\beta^{n}$, at the expense of a higher protection $\theta^{d}$ in order to compensate an honest inventor. A higher $\alpha$ makes it more costly to deter opportunistic patenting, and it may not be optimal to do so. ${ }^{49}$

Proposition 9. (Limits of private bounty). When there is type II error, (i) the deterrence bounty is not feasible if condition (15) holds; and (ii) even feasible, it is not optimal to deter opportunistic patenting with bounties if the quality of issued patents $(\alpha)$ is not too low.

Public bounty: Lastly, let us consider a bounty financed by public funds. To keep consistency, public funds are assumed available to reward the innovation as well, i.e., a prize scheme is also included. We compare different policy combinations: Using the public fund only to encourage innovation (a research grant), only to encourage patent challenge (a public bounty), to do both, or none. ${ }^{50}$

In general, public funds also entail social cost. Instead of the standard shadow cost of tax collection, we resort to the patent authority's information constraint. Suppose

\footnotetext{
${ }^{48}$ The existence of optimal $b^{d}>0$ is guaranteed by the property of cost function $c$, especially $c^{\prime}(1)=\infty$.

${ }^{49} \mathrm{It}$ is easy to confirm this point with a two-point searching technology, i.e. $B$ has an exogenous probability $\beta$ to find the prior art by incurring a fixed search cost.

${ }^{50}$ We don't let the patent authority mix the two regimes at the same stage, e.g., we don't allow the patent authority to reward an inventor partly by patent and partly by a prize. Given the model's linear structure this should not be a restriction.
} 
that there is a mass $M$ of rent-seekers, who can 'attack' a prize or public bounty system by producing fake patent applications and defeating prior arts with a very small cost $\delta>0$. By a fake patent application, we mean one seeks to patent an invention that has no value, and there exists evidence to show that it doesn't meet the patentability criteria. Suppose $A$ is not among those rent-seekers, ${ }^{51}$ but the patent authority cannot distinguish between a patent application from $A$ or a rent-seeker.

Consider our basic model, the case of multiple-user with single searcher. For simplicity, we ignore the search cost and fix the search intensity $\beta$. Assume that the policy cannot depend on the volume of patent applications or challenges. ${ }^{52}$ When patents are the only policy tool, the patent authority's optimization program is $(\mathcal{P})^{s}$ in Section 3 , with $c=0$ and $\beta$ fixed. Absent search cost, the optimal re-allocation is governed by collusion concerns (regime (ii)). The patent-holder gets a patent with $\theta^{*}$ when no challenge, and the challenger gets $\lambda \theta^{*}$ by submitting the prior art. Since a patent is useless to them, no rent-seekers will spend $\delta$ to produce fake applications. When public funds are introduced, consider where the money is spent:

Challenge only (a public bounty but not a prize system): To overcome the collusion problem the bounty should be at least $\lambda \theta^{*} S$. The patent is entirely invalidated after the challenge, and full production surplus $V^{e}$ is realized. But a positive bounty attracts rent-seekers. Each of them incurs $\delta$ to apply for a patent and then invalidates it, with social welfare

$$
\begin{aligned}
\mathcal{W}^{c} & =\alpha\left(V^{e}-\theta^{*} D-K\right)+(1-\alpha)\left[(1-\beta)\left(V^{e}-\theta^{*} D\right)+\beta V^{e}\right]-\delta M \\
& =V^{e}-[\alpha+(1-\alpha)(1-\beta)] \theta^{*} D-\alpha K-\delta M ;
\end{aligned}
$$

Innovation plus challenge : If no patent rights are granted, there is no efficiency loss on production. To encourage innovation, the research prize should be at least $K>0$; and to encourage challenge, the bounty should be at least as large as the research prize. ${ }^{53}$ Rent-seekers rush to the patent office to win the higher of these two.

\footnotetext{
${ }^{51}$ Alternatively, we can assume that $A$ has the ability to produce the bogus and useless patent, but this activity doesn't crowd out her effort to spend $k$ and bring the useful innovation.

${ }^{52}$ Since the patent authority cannot discriminate a useful invention from a fake invention, doing so simply dilutes the reward to a true inventor.

${ }^{53}$ Note that a challenge doesn't improve social welfare here, for, again, we've assumed away the incentive effect of the challenge on innovation. Instead, if $A$ chooses between a research project requiring cost $K$, or applying for a prize on an existing technology (with zero cost) and risk the challenge, then to encourage innovation the authority cannot simply distribute money to any applicant. The challenge is necessary to
} 
Social welfare is

$$
\mathcal{W}^{i+c}=\alpha\left(V^{e}-K\right)+(1-\alpha) V^{e}-\delta M=V^{e}-\alpha K-\delta M
$$

Innovation only : Suppose the patent authority rewards innovation with a prize $b^{p} \geq K$, and encourages prior art submission with collusion-deterrence patent rights $\theta^{p}$. Rent-seekers incur $\delta M$ to get the prize $b^{p}$, but will not challenge it. Social welfare is

$$
\begin{aligned}
\mathcal{W}^{i} & =\alpha\left(V^{e}-K\right)+(1-\alpha)\left[(1-\beta) V^{e}+\beta\left(V^{e}-\theta^{p}(N-1) d\right)\right]-\delta M \\
& =V^{e}-(1-\alpha) \beta \theta^{p}(N-1) d-\alpha K-\delta M
\end{aligned}
$$

It is not surprising to see that public funds should not be used under heavy rentseeking activity. For $M$ large enough, a pure patent system is superior to alternatives incorporating public funds. A more interesting point is that, a pure reward scheme dominates the other two mixed regimes: $\mathcal{W}^{i+c}$ is greater than $\mathcal{W}^{i}$ and $\mathcal{W}^{c}$. This implies that, if we don't want to abandon the patent system and switch to the prize system, probably we should not adopt the public bounty either.

Proposition 10. (Public bounty). The effectiveness of using public funds in the innovation policy is constrained by the rent-seeking problem. And a pure public bounty is not optimal.

Remark. Here using public funds only entails a 'fixed cost' $\delta M$. Therefor, once this cost is incurred, the full advantage of public funds should be exhausted, and there is no point to mix it with the patent system and generate additional social loss. Alternatively, a 'variable cost' component presents if there is another group of heterogeneous rentseekers, each has different costs to fabricate the fake invention. The social cost of public fund then is increasing in the size of monetary reward because a higher reward attracts more rent-seekers. This makes public bounty more attractive than a research prize, for to deter collusion it suffices to post a bounty of $\lambda \theta^{*} S<\theta^{*}(N-1) \pi<K$. Nevertheless, our result holds as long as the fixed cost component is large enough. ॥

overcome $A$ 's moral hazard problem. With probability $\beta$ and a prize $R$, the incentive constraint is

$$
R-K \geq(1-\beta) R \Rightarrow \beta R \geq K .
$$

Without challenge $(\beta=0)$, this constraint cannot be satisfied. 


\section{References}

Caillaud, B. and A. Duchêne, (2005), "Patent Office in Innovation Policy: Nobody's Perfect," working paper.

Chiou, J.-Y., (2008), "The Patent Quality Control Process: Can We Afford (an) Rationally Ignorant Patent Office?" working paper.

Choi, J. P., (2005), "Live and Let Live: A Tale of Weak Patents," Journal of European Economic Association, 3(2-3): 724-33.

Farrell, J. and R. Merges, (2004), "Incentives to Challenge and Defent Patents: Why Litigation Won't Reliably Fix Patent Office Errors and Why Admonistrative Patent Review Might Help.” Berkeley Technology Law Journal, 19(1):

Farrell, J. and C. Shapiro, (2008), "How Strong Are Weak Patents?" American Economic Review, 98(4): 1347-69.

Federal Trade Commission, (2003), To Promote Innovation: A Proper Balance of Competition and Patnet Law and Policy.

Graham, S., B. Hall, D. Harhoff and D. Mowery, (2003), "Post-Issue Patent Quality Control: A Comparative Study of U.S. Patent Re-Examinations and European Patent Oppositions," Patents in the Knowledge-Based Economy, W. Cohen and S. Merrill eds., Washington, D.C.: the National Academies Press.

Graham, S. and D. Harhoff, (2005), "Would the U.S. Benefit from Patent Post-Grant Reviews? Evidence from a 'Twinning' Study," working paper.

Green, J. and S. Scotchmer, (1995), "On the division of Profit in Sequential Innovation," RAND Journal of Economics, 26(1): 20-33.

Hall, B. and D. Harhoff, (2004), "Post-Grant Reviews in the U.S. Patent System-Design Choices and Expected Impact," Berkeley Technology Law Journal, 19(3): 989-1015.

Hall, B., and R. Ziedonis, (2001), "The Patent Paradox Revisited: An Empirical Study of Patenting in the U.S. Semiconductor Industry, 1979-1995," RAND Journal of Economics, 32(1): 101-128. 
Hovenkamp, H., M. Janis, and M. Lemley, (2003), "Anticompetitive Settlement of Intellectual Property Disputes," Minnesota Law Review, 87(6): 1719-61.

Jaffe, A. and J. Lerner, (2004), Innovation and its Discontents: How Our Broken Patent System is Endangering Innovation and Progress, and What to Do About it, Princeton University Press.

Khalil, F. and J. Lawarrée, (2006), "Incentives for Corruptible Auditors in the Absence of Commitment," Journal of Industrial Economics, 54(2): 269-91.

Laffont, J.-J. and D. Martimort, (1999), "Separation of Regulators against Collusive Behavior," RAND Journal of Economics, 30(2): 232-62.

Lemley, M., (2001), "Rational Ignorance at the Patent Office," Northwestern University Law Review, 95(4): 1495-1529.

Levin, J. and R. Levin, (2003), "Benefits and Costs of an Opposition Process," Patents in the Knowledge-Based Economy, W. Cohen and S. Merrill, eds., Washington, D.C.: the National Academies Press.

Miller, J., (2003), "Building a Better Bounty: Litigation-Stage Rewards for Defeating Patents," Berkeley Technology Law Review, 19(2): 668-741.

National Academies of Science, (2004), A Patent System for the 21st Century.

Nordhaus, W., (1969), Invention, Growth, and Welfar: A Theoretical Treatment of Technological Change, Cambridge, MA: MIT Press.

Quillen, C. and O. Webster, (2001), "Continuing Patent Applications and Performance of the U.S. Patent Office," Federal Circuit Bar Journal, 11(1): 1-21.

Thomas, J. (2001), "Collusion and Collective Action in the Patent System: A Proposal for Patent Bounties," Univeristy of Illinois Law Review, 2001 (1): 305-53.

Tirole, J. (1986), "Hierarchies and Bureaucracies: On the Role of Collusion in Organization," Journal of Law, Economics, and Organization, 2: 181-214. 